\title{
Larvas veliger de gastrópodos Prosobranchia provenientes de Punta de Lobos, Cuarta Región, Chile
}

\author{
Veliger larvae of Prosobranchia gastropods from Punta de Lobos, \\ Cuarta Región, Chile
}

\begin{abstract}
M. SOLEDAD ROMERO \& EUGENIA L. VALDEBENITO
Departamento de Biología Marina, Facultad de Ciencias del Mar, Universidad Católica del Norte, Casilla 117, Coquimbo, Chile; e-mail:msromero@nevados.ucn.cl
\end{abstract}

\begin{abstract}
RESUMEN
El presente estudio constituye un registro descriptivo e ilustrado de larvas de gastrópodos presentes a $2,8 \mathrm{~km}$ de la costa frente a Punta de Lobos ( $30^{\circ} \mathrm{S}, 71^{\circ} 21^{\prime}$ O), Chile, centro-norte, en el período marzo-diciembre de 2000. Las muestras fueron recolectadas con malla de $300 \mu \mathrm{m}$ hasta los $50 \mathrm{~m}$ de profundidad. Se determinaron 19 especies larvales de las cuales 13 se describen aquí. Se identificaron larvas de Turritella cingulata Sowerby, 1833, Crassilabrum crassilabrum (Sowerby, 1834), Xanthochorus cassidiformis (Blainville, 1832), Nassarius dentifer (Powys, 1835) y Salitra radwini Marincovich, 1973. Las restantes especies se asignaron a género o familia. Se entrega una caracterización morfológica mediante microscopía fotónica y electrónica de barrido y se informa la ocurrencia por número y especie de las larvas recolectadas.
\end{abstract}

Palabras clave: larvas, veliger, Prosobranchia, Cuarta Región, Chile.

\begin{abstract}
The present study constitutes a descriptive and illustrated record of gastropod larvae present at $2.8 \mathrm{~km}$ off "Punta de Lobos" ( $\left.30^{\circ} \mathrm{S}, 71^{\circ} 21^{\prime} \mathrm{W}\right)$, north-central Chile, in the period March- December of 2000. Samples were collected with a $300 \mu \mathrm{m}$ mesh down to $50 \mathrm{~m}$ of depth. The larvae of 19 species were determined, of which 13 are described here. Larvae of Turritella cingulata Sowerby, 1833, Crassilabrum crassilabrum (Sowerby, 1834), Xanthochorus cassidiformis (Blainville, 1832), Nassarius dentifer (Powys, 1835) and Salitra radwini Marincovich, 1973 were identified. The remaining species were assigned to genus or family. The larvae were characterized with the aid of light and scanning electron microscopy and we report the occurrence by number and species of collected larvae.
\end{abstract}

Key words: larvae, veliger, Prosobranchia, Cuarta Región, Chile.

\section{INTRODUCCIÓN}

La identificación específica de larvas de gastrópodos contribuye al conocimiento de numerosos aspectos de la biología de estos moluscos. Considerando que los gastrópodos se encuentran en numerosos y variados tipos de hábitat, la determinación de la ocurrencia espacial y temporal de sus larvas permitiría establecer patrones ecológicos, proyectar valores de reclutamiento, estimar biodiversidad, su contribución en tramas tróficas, al flujo génico de poblaciones independientes, analizar relaciones evolutivas, paleoecológicas y otros (Thorson 1946, 1950, Marwick 1957, Thiriot-Quiévreux 1968, 1970, 1983, Scheltema 1971, 1986, Taylor 1975, Jung 1975, Marshall 1978, 1983, Jablonski \& Lutz 1983, Scheltema \& Williams 1983, Colman et al. 1986, Allmon 1988, 1996, Lima \& Lutz 1990,
Kool 1993, Acevedo 1995, Kowalke \& Bandel 1996, Bandel et al. 1997, Bandel \& Kowalke 1997a, 1997b, Nützel \& Kießling 1997, Kowalke 1998a, 1998b, 2001).

A pesar de que Chile cuenta con una costa extensa, que abarca una amplia variedad de regiones climáticas y zoogeográficas (Lancelloti \& Vásquez 2000), los estudios descriptivos y/o taxonómicos de larvas planctónicas de gastrópodos son escasos (Gallardo 1973, 1977, 1981, Gallardo \& González 1984, DiSalvo 1988, Acevedo 1995), lo que no permite analizar o relacionar aspectos como los mencionados anteriormente.

La ornamentación de la protoconcha larval se ha interpretado de diversas maneras: como carácter ancestral (Marwick 1957, Marshall 1978, 1983, Allmon 1988, 1996, Kowalke 1998a, 1998b, Nützel 1998, Hickman 1999, 2001), como adapta- 
ción para la existencia planctónica, o como artefacto producido por biomineralización temprana (Hickman 2001). De cualquier modo, la ornamentación de la concha larval se reconoce como un carácter de gran valor taxonómico y la identificación de las larvas de gastrópodos se puede lograr a través de cuatro métodos: (1) observación de larvas cultivadas a partir de individuos parentales conocidos (Lebour 1933b, 1934b, 1935a, 1935b, 1936, 1937, 1945, Fretter \& Graham 1962, Scheltema 1962, Thorson 1965, Thiriot-Quiévreux 1967a, 1967b, 1969, 1977, Struhsaker \& Costlow 1968, Robertson 1974, Thiriot-Quiévreux \& Scheltema 1982, Scheltema \& Williams 1983, DiSalvo 1988, Romero 1995), (2) inducción de la metamorfosis de larvas obtenidas del plancton y seguimiento de su desarrollo hasta la aparición de juveniles o adultos identificables (Lebour 1931a, 1931b, 1932, 1933a, 1933b, 1933c, 1934a, 1934b, 1937, 1945, Thorson 1946, Thiriot-Quiévreux 1967a, 1967b, 1975, 1980, Taylor 1975), (3) comparación de la morfología de larvas recolectadas con la de otras especies identificadas por alguno de los métodos anteriores (Robertson 1971, Rodríguez \& Thiriot-Quiévreux 1974, Jung 1975, Thiriot-Quiévreux \& Rodríguez 1975, ThiriotQuiévreux 1980, 1983, Thiriot-Quiévreux \& Scheltema 1982, Scheltema \& Williams 1983), y (4) comparar la morfología de las conchas larvales con el ápice de ejemplares adultos identificados y bien conservados (Marwick 1957, Scheltema 1971, Rodríguez \& Thiriot-Quiévreux 1974, 1975, Thiriot-Quiévreux \& Rodríguez 1975, Seapy
\&Thiriot-Quiévreux 1994, Kowalke \& Bandel 1996, Bandel \& Kowalke 1997 ab, Nützel \& Kießling 1997, Kowalke 1998ab, 2001, Marshall 1978,1983)

En este tipo de estudios el microscopio electrónico de barrido (MEB) constituye una herramienta insustituible. Debido a sus características ópticas superiores de magnificación, profundidad de campo y resolución, el MEB permite caracterizar detalladamente la morfología de conchas larvales facilitando la identificación taxonómica (Robertson 1971, Thiriot-Quiévreux 1972).

El presente estudio constituye un catastro de larvas de gastrópodos presentes en Punta de Lobos, en la costa centro-norte de Chile, en el período marzo-diciembre de 2000. El objetivo de este trabajo es entregar una caracterización morfológica mediante microscopía fotónica y electrónica de barrido de las larvas de 13 especies e informar acerca de sus abundancias relativas en muestras de plancton.

\section{MATERIALES Y MÉTODOS}

Las larvas fueron obtenidas a $2,8 \mathrm{~km}$ de la costa frente a Punta de Lobos $\left(30^{\circ} \mathrm{S}, 71^{\circ} 21^{\prime} \mathrm{O}\right) \mathrm{O}$ diante muestreos planctónicos realizados entre el 1 de marzo y el 25 de diciembre de 2000 , en las fechas indicadas en la Tabla 1. El método de muestreo consistió en un lance doble oblicuo, hasta $\operatorname{los} 50 \mathrm{~m}$ de profundidad, utilizando red bongo de $60 \mathrm{~cm}$ de diámetro de boca, con malla de

TABLA 1

Porcentaje de larvas capturadas en cada fecha de muestreo en Punta de Lobos, Cuarta Región; n corresponde al número total de larvas

Percentage of larvae captured in every date of sampling in Punta de Lobos, Cuarta Region; $\mathrm{n}$ corresponds to the total number of larvae

\begin{tabular}{|c|c|c|c|c|c|c|c|c|c|}
\hline Especie & $\mathrm{n}$ & $\begin{array}{c}\text { Marzo } \\
1\end{array}$ & $\begin{array}{c}\text { Marzo } \\
31\end{array}$ & $\begin{array}{c}\text { Abril } \\
3\end{array}$ & $\begin{array}{c}\text { Mayo } \\
30\end{array}$ & $\begin{array}{l}\text { Agosto } \\
2\end{array}$ & $\begin{array}{l}\text { Agosto } \\
22\end{array}$ & $\begin{array}{c}\text { Noviembre } \\
24\end{array}$ & $\begin{array}{c}\text { Diciembre } \\
25\end{array}$ \\
\hline Turritella cingulata & 17 & 88,2 & 5,9 & 0 & 5,9 & 0 & 0 & 0 & 0 \\
\hline Cerithiidae & 107 & - & 5,61 & 0,93 & 14,02 & 38,32 & 15,88 & 17,76 & 7,48 \\
\hline Cerithiopsis sp. & 13 & - & 7,7 & 0 & 38,5 & 15,4 & 15,4 & 15,4 & 7,7 \\
\hline Triphora sp. & 6 & - & 16,7 & 0 & 50 & 16,7 & 16,7 & 0 & 0 \\
\hline Triphorinae & 6 & - & 16,67 & - & 50,00 & - & 16,67 & 16,67 & - \\
\hline Janthina sp. & 5 & 20 & 20 & - & 40 & - & - & - & 20,00 \\
\hline Littorinidae & 13 & 7,69 & - & - & 30,77 & - & 15,38 & - & 46,15 \\
\hline Crassilabrum crassilabrum & 28 & 7,14 & 7,14 & 14,29 & 53,57 & 14,29 & - & - & 3,57 \\
\hline Xanthochorus cassidiformis & 10 & 50,00 & - & 40,00 & - & - & - & - & 10,00 \\
\hline Salitra radwini & 4 & 2,78 & 16,67 & 8,33 & 25,93 & 12,04 & 6,48 & 25,93 & 1,85 \\
\hline Mitrella sp. & 8 & 50,00 & - & - & 25,00 & 25,00 & - & - & - \\
\hline Nassarius dentifer & 52 & 17,31 & - & 1,92 & 1,92 & - & - & - & 78,85 \\
\hline Nassarius sp. & 29 & 55,17 & 13,79 & 3,45 & 3,45 & - & - & - & 24,14 \\
\hline
\end{tabular}


$300 \mu \mathrm{m}$ de apertura de poro. Las muestras completas de plancton vivo fueron diluidas a $10 \mathrm{~L}$ en contenedores plásticos y transportadas al laboratorio. Para obtener las larvas se agitó vigorosamente la columna de agua con el fin de inducir la retracción de las larvas en sus conchas y su posterior decantación. Luego se revolvió el agua con movimientos circulares para concentrar los organismos más pesados en el centro del fondo y se succionaron con un tubo de vidrio de $1 \mathrm{~cm}$ de diámetro. El procedimiento se repitió hasta no obtener ninguna larva en seis a diez extracciones sucesivas. El plancton extraído fue observado

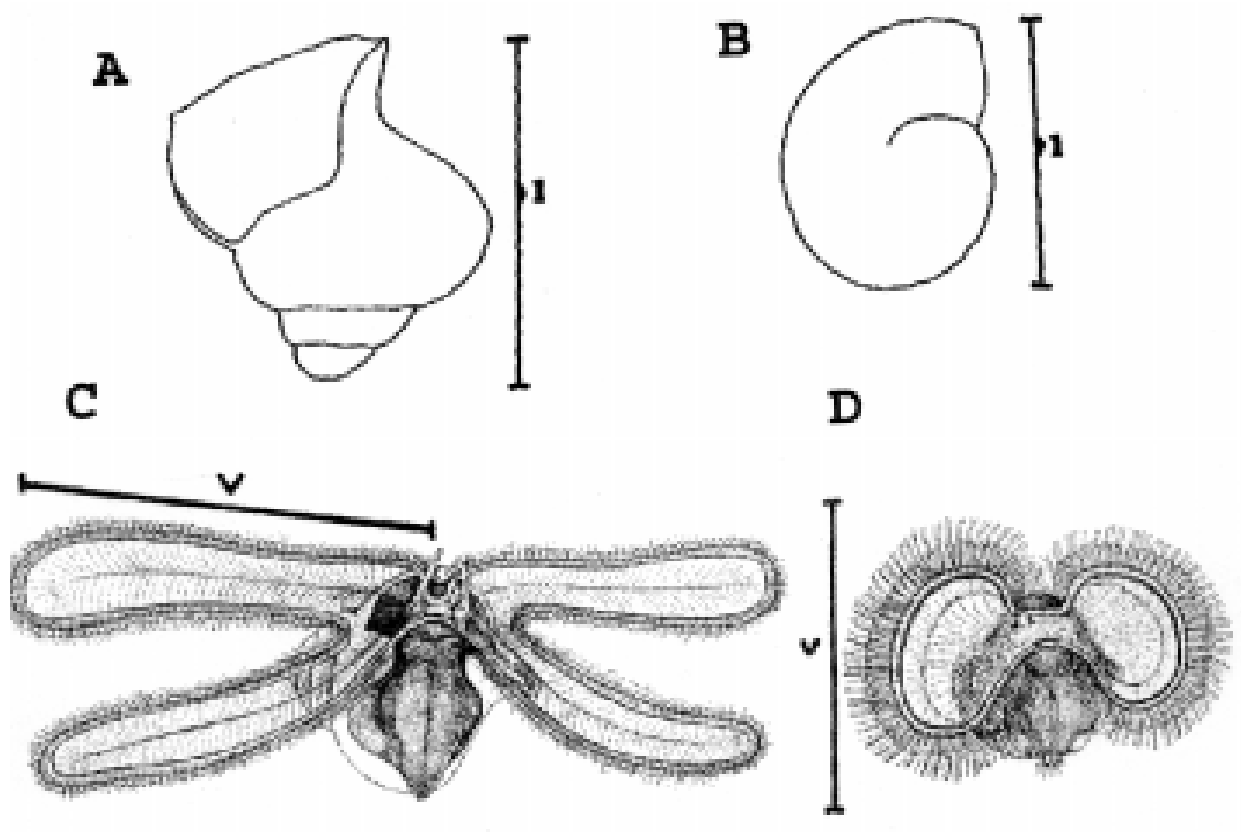

$\mathbf{E}$

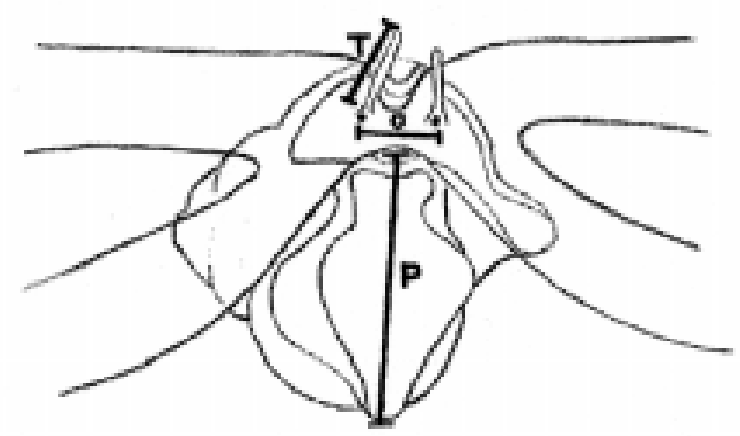

Fig. 1: Dimensiones morfométricas de estructuras larvales: (A) longitud máxima de la concha larval; (B) longitud máxima de la protoconcha embrionaria; (C) longitud del lóbulo velar en larvas tetralobuladas; (D) longitud del lóbulo velar en larvas bilobuladas; (E) longitud de tentáculos (t) pie (p) y distancia entre los ojos (o).

Morphometric dimensions of larval structures: (A) Length maximum of larval shell; (B) maximum length of embryonic shell; (C) length of velar lobe in four-lobed larvae; (D) length of velar lobe in bilobed larvae; (E) tentacles (t) and foot (p) length and distance between eyes (o). 
Para describir la morfología de las conchas larvales, las larvas fueron muertas con una gota de glutaraldehído y tratadas con una solución de hipoclorito de sodio comercial en una concentración final de 1,6\%. La eliminación de materia orgánica fue observada bajo lupa. Las muestras fueron secadas a $60^{\circ} \mathrm{C}$ por 4 a $8 \mathrm{~h}$, se montaron en portaobjetos de bronce y finalmente se cubrieron con oro siendo observadas y fotografiadas en un microscopio electrónico de barrido JEOL T300. Se midió la longitud máxima, esto es desde el ápice al extremo del canal sifonal o en su defecto, al borde de la apertura (Fig. 1A). Las protoconchas embrionarias se midieron desde el extremo de la apertura hasta el extremo opuesto más lejano de la misma protoconcha (Fig. 1B).

Para describir la morfometría de las partes blandas, las larvas vivas fueron observadas en un microscopio Nikon OPTIPHOT y fotografiadas con una cámara Nikon FX 35A. Las mediciones de partes blandas corresponden a diámetro (Fig. 1C) o longitud de lóbulos velares (Fig. 1D), longitud de los tentáculos, distancia entre los ojos y longitud del pie considerado desde la boca al extremo del metapodio (Fig. 1E). No se midió a partir del propodio debido a que esta porción del pie es constantemente elongada y contraída en larvas competentes. Debido al bajo número de larvas de algunas especies, se realizaron mediciones en un solo ejemplar. Estos casos se indican en el texto.

La metamorfosis de algunos ejemplares se indujo ofreciendo trozos de sustrato (piedras, cirripedios) en los mismos contenedores de cultivo.

\section{RESULTADOS}

Caenogastropoda-Neotaenioglossa: Turritella cingulata Sowerby, 1833 (Turritellidae)

La protoconcha larval es espiral cónica, dextrógira, color ámbar, compuesta por 3,5 anfractos, mide $457 \mu \mathrm{m}$ de longitud promedio $(\mathrm{DE}=90,8 ; \mathrm{n}=9)$. En vista ventral, la apertura es redondeada, el borde externo es recto y suavemente festoneado (Fig. 2A). El segundo anfracto y la espira del cuerpo, presentan ornamentación compuesta de gránulos que tienden a una ordenación lineal formando bandas (Fig. 2B). Todos los anfractos presentan líneas axiales finas que corresponderían a estrías de crecimiento. La protoconcha embrionaria mide $130 \mu \mathrm{m}(\mathrm{DE}=$ $11,1 ; \mathrm{n}=4)$, presenta una ornamentación compuesta de relieves irregulares que le otorgan una apariencia rugosa (Fig. 2C).
Las partes blandas (Fig. 2D) se caracterizan como sigue: el velo es bilobulado y los lóbulos son redondos y asimétricos. El lóbulo izquierdo mide $219 \mu \mathrm{m}$ de diámetro $(\mathrm{DE}=56, \mathrm{n}=8)$ y el derecho $183 \mu \mathrm{m}(\mathrm{DE}=36,2 ; \mathrm{n}=8)$. Los tentáculos son simétricos, miden $65 \mu \mathrm{m}$ de longitud (DE $=8, \mathrm{n}=9$ ). El pie es redondeado, despigmentado, mide $255 \mu \mathrm{m}$ de longitud $(\mathrm{DE}=25, \mathrm{n}=4)$.

Esta especie fue identificada como Turritella cingulata en base a la observación de larvas desarrolladas a partir de posturas de $T$. cingulata (Romero et al. en prensa). Además, tres larvas capturadas el 1 de marzo metamorfosearon en laboratorio 57 días después, alcanzando 1.042 $\mu \mathrm{m}$ de longitud máxima ( $\mathrm{DE}=200,5 ; \mathrm{n}=3$ ) y el aspecto reconocible de un adulto a las 10 semanas post-metamorfosis.

Larvas de T. cingulata fueron abundantes sólo en el muestreo del 1 de marzo, en los dos meses posteriores se capturó sólo una larva por muestra (Tabla 1).

\section{Caenogastropoda-Neotaenioglossa: Cerithiidae?}

La protoconcha larval es espiral cónica, dextrógira, color ámbar, formada por 4,25 anfractos, la longitud máxima promedio es de 642 $\mu \mathrm{m}(\mathrm{DE}=47,1 ; \mathrm{n}=6)$. La apertura es redonda y presenta un canal sifonal corto (Fig. 3A). En vista ventral, el anfracto del cuerpo se divide en dos mitades. La mitad abapical es lisa y la mitad adapical presenta una ornamentación compuesta por costillas axiales y dos a cuatro líneas espirales formadas por gránulos finos (Fig. 3A y 3B). La protoconcha embrionaria es globular, ornamentada con gránulos gruesos distribuidos uniformemente (Fig. 3C). La protoconcha embrionaria midió $127,5 \mu \mathrm{m}$ en un ejemplar.

Las partes blandas (Fig. 3D) se caracterizan como sigue: el velo es bilobulado y los lóbulos son redondeados y asimétricos. El lóbulo derecho mide $367 \mu \mathrm{m}$ de diámetro promedio $(\mathrm{DE}=51 ; \mathrm{n}$ =6), el izquierdo mide $263 \mu \mathrm{m}(\mathrm{DE}=37,8 ; \mathrm{n}=6)$. Los ojos son negros, se ubican al lado de cada tentáculo, con una distancia promedio de $137 \mu \mathrm{m}$ $(\mathrm{DE}=12,1 ; \mathrm{n}=6)$ entre ambos. Los tentáculos son cortos, simétricos, miden $97 \mu \mathrm{m}$ promedio de longitud $(\mathrm{DE}=8,2 ; \mathrm{n}=6)$. El pie mide $292 \mu \mathrm{m}$ promedio $(\mathrm{DE}=31,3 ; \mathrm{n}=6)$, redondeado con la región metapodial de forma triangular, cristalino excepto en la región superior adyacente a la boca donde esta pigmentado negro. La pigmentación abarca la región cefálica.

Cerithiidae fue más frecuente en los muestreos realizados en agosto y noviembre constituyendo el 38 y $17,8 \%$ del total de la muestra, respectiva- 
mente. En otros meses su ocurrencia fue escasa o ausente. Esta especie fue la más abundante en el total del período muestreado (Tabla 1).

Caenogastropoda-Neotaenioglossa: Cerithiopsis sp. (Cerithiopsidae)

La protoconcha larval es espiral cónica, dextrógira, color café, formada por 3,25 anfractos suavemente convexos. La longitud máxima promedio es de $549 \mu \mathrm{m}(\mathrm{DE}=9, \mathrm{n}=7)$. La apertura presenta un pico ensanchado en el extremo. No presenta canal sifonal (Fig. 4A). Los anfractos están ornamentados con líneas formadas por gránulos ortoclinas en la región dorsal y opistoclinas en la región ventral (Fig. 4B). La protoconcha embrionaria esta ornamentada con gránulos ordenados formando bandas espirales y mide $123 \mu \mathrm{m}$ $(\mathrm{DE}=16,1 ; \mathrm{n}=2)$ (Fig. 4C)
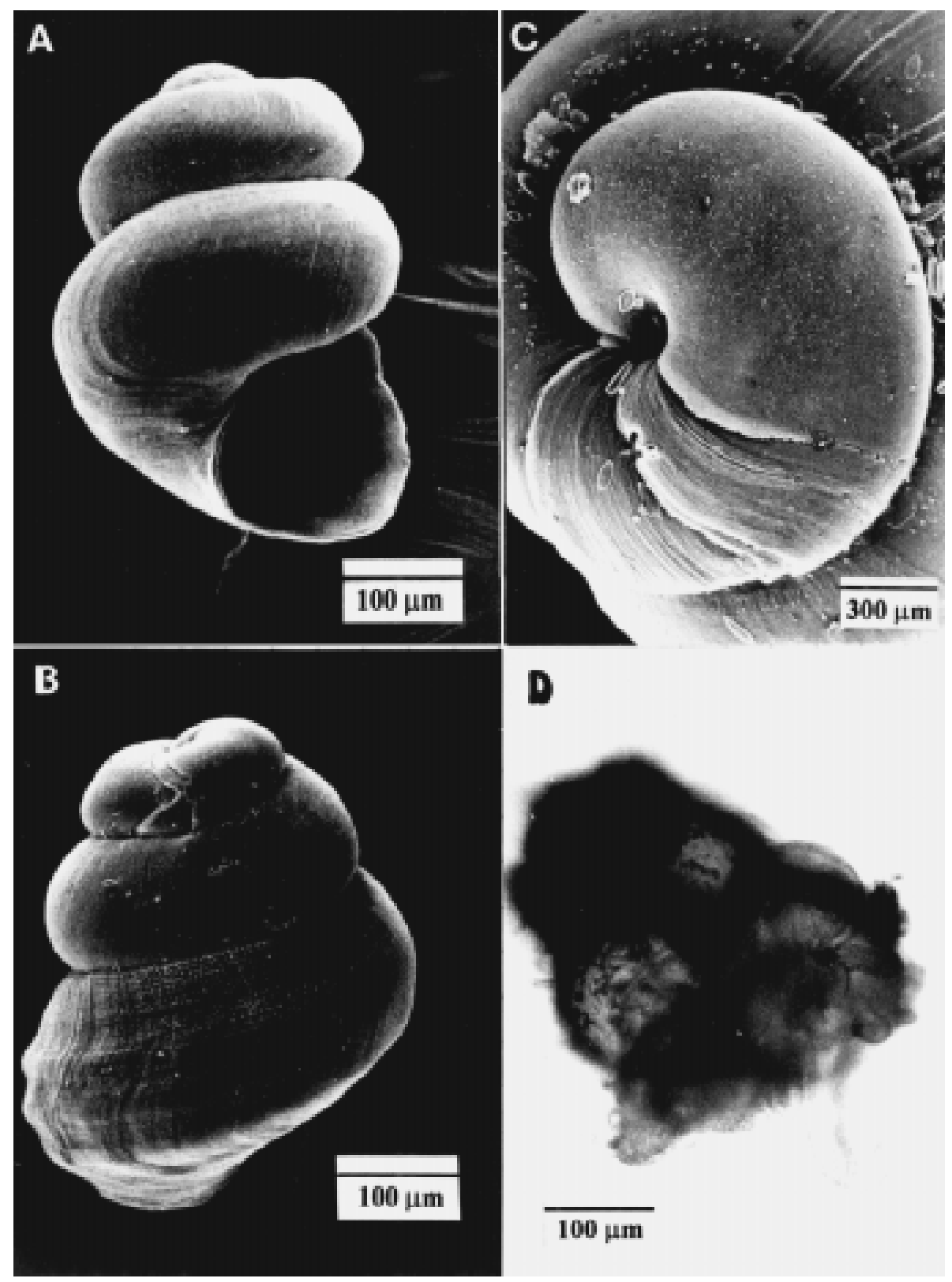

D

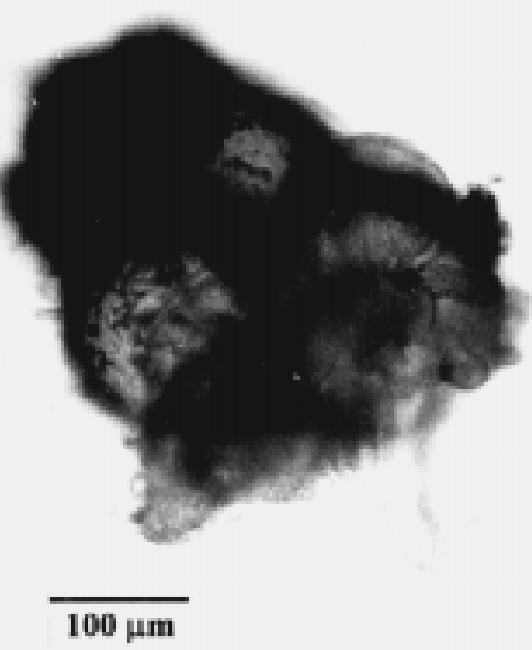

Fig. 2: Turritella cingulata: (A) vista ventral de la protoconcha larval; (B) vista dorsal de la protoconcha larval; (C) protoconcha embrionaria; (D) larva viva en vista frontal.

Turritella cingulata: (A) ventral view of larval protoconch; (B) dorsal view of larval protoconch; (C) embryonic protoconch; (D) frontal view of a living larva. 
Las partes blandas (Fig. 4D) se caracterizan como sigue: el velo es bilobulado, los lóbulos son despigmentados y asimétricos. El lóbulo derecho es ovalado, mide $307 \mu \mathrm{m}$ de longitud ( $\mathrm{DE}=18,9$; $\mathrm{n}=7$ ), el lóbulo izquierdo es redondeado y mide $203 \mu \mathrm{m}(\mathrm{DE}=7,6 ; \mathrm{n}=7)$. Los tentáculos miden 87 $\mu \mathrm{m}$ de longitud promedio $(\mathrm{DE}=18,9 ; \mathrm{n}=7)$, son simétricos y se encuentran curvados bajo el pico sobresaliendo en los extremos. Los ojos son negros, están ubicados en la base de los tentáculos y se encuentran a una distancia de $111 \mu \mathrm{m}$ entre ambos $(\mathrm{DE}=8,9 ; \mathrm{n}=7)$. El pie mide $254 \mu \mathrm{m}$ de longitud ( $\mathrm{DE}=36, \mathrm{n}=7$ ), es redondeado, levemente triangular en la región del metapodio, presenta manchas pigmentadas de color negro en su superficie.

Esta especie fue asignada al género Cerithiopsis Forbes \& Hanley, 1850 en base a la descripción realizada por Marshall (1978) y Nützel (1998).

Esta especie fue poco frecuente o ausente en los tres primeros muestreos, sin embargo, en el
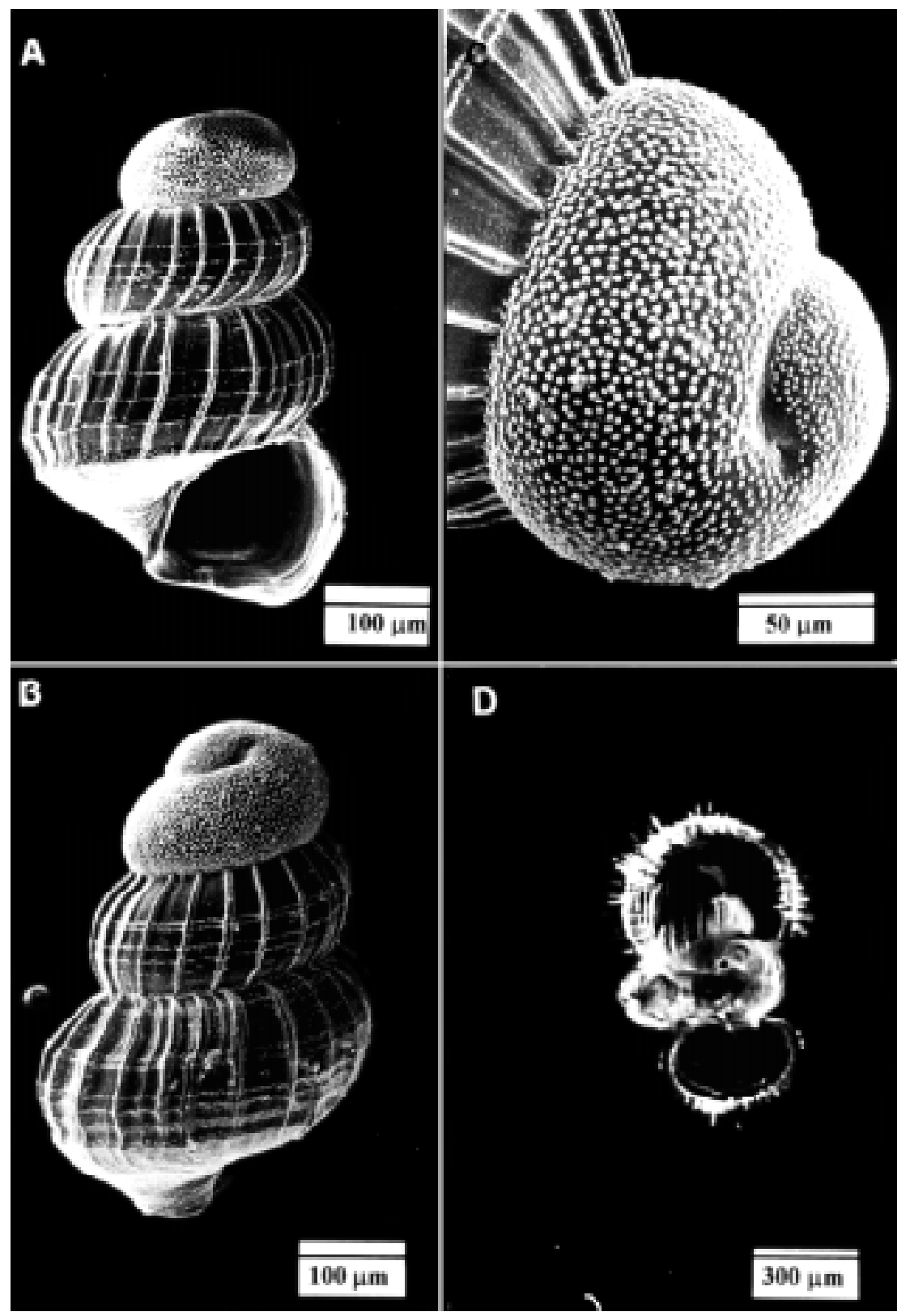

Fig. 3: Cerithidae: (A) vista ventral de la protoconcha larval; (B) vista dorsal de la protoconcha larval; (C) protoconcha embrionaria; (D) larva viva en vista frontal.

Cerithidae: (A) ventral view of larval protoconch; (B) dorsal view of larval protoconch; (C) embryonic protoconch; (D) frontal view of a living larva. 
muestreo del 30 de mayo se capturó un número mayor de ejemplares, correspondiendo al $58 \%$ del total de los muestreos (Tabla 1).

Caenogastropoda-Neotaenioglossa: Triphora sp. (Triphoridae)

La protoconcha larval es espiral cónica, levógira, de color castaño rojizo, formada por 3,25 anfractos. La longitud máxima promedio es de
$570 \mu \mathrm{m}(\mathrm{DE}=69,8 ; \mathrm{n}=4)$. La protoconcha con máximo desarrollo midió $630 \mu \mathrm{m}$ de largo. Todos los anfractos presentan costillas axiales atravesadas por un relieve espiral en el segundo anfracto y por dos, en el anfracto del cuerpo. En vista ventral, la mitad abapical del anfracto del cuerpo es lisa, excepto en la región adyacente al hombro donde está ornamentada con una banda cuadriculada. La apertura es redonda y posee un pico corto en la mitad del anfracto (Fig. 5A). No presenta canal sifonal. La protoconcha embrionaria es glo-
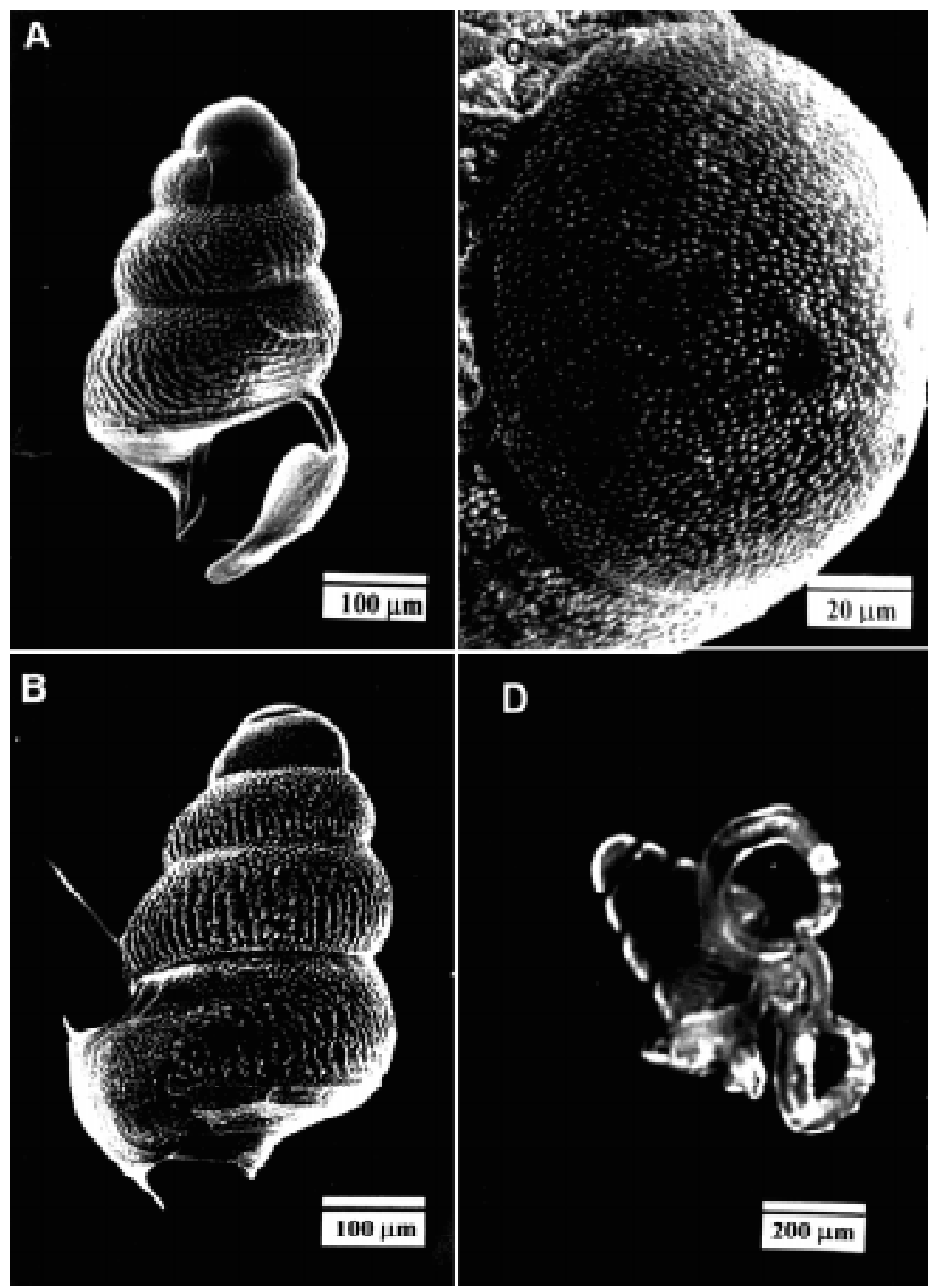
bular, ornamentada con gránulos gruesos que forman bandas poco definidas (Fig. 5B). El diámetro de la protoconcha embrionaria, medido en un solo ejemplar fue de $137,3 \mu \mathrm{m}$.

Las partes blandas (Fig. 5C) se caracterizan como sigue: el velo es bilobulado, los lóbulos son despigmentados y asimétricos. El lóbulo derecho es más grande que el izquierdo, ovalado, mide $475 \mu \mathrm{m}(\mathrm{DE}=106, \mathrm{n}=2)$ y sobrepasa el ápice de la concha. El izquierdo es redondeado, mide 215 $\mu \mathrm{m}(\mathrm{DE}=21, \mathrm{n}=2)$. Los tentáculos son simétricos, transparentes, curvados hacia adelante, miden $6,5 \mu \mathrm{m}$ de longitud ( $\mathrm{DE}=7, \mathrm{n}=2$ ). Los ojos

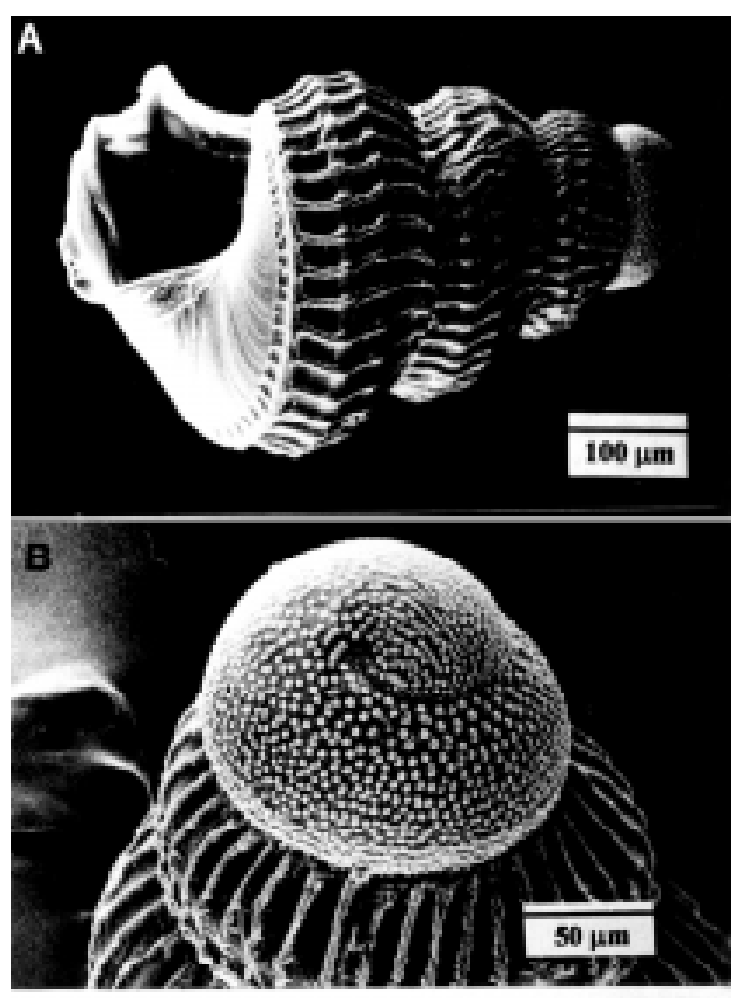

c

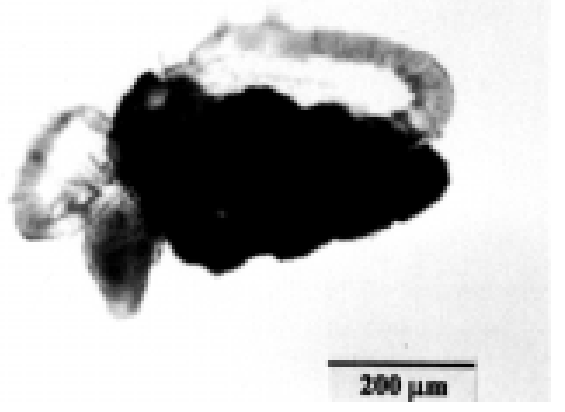

Fig. 5: Triphoridae: (A) vista ventral de la protoconcha larval; (B) protoconcha embrionaria; (C) larva viva en vista frontal.

Triphoridae: (A) ventral view of larval protoconch; (B) embryonic protoconch; (C) frontal view of a living larva. son negros, se ubican en la base de los tentáculos a una distancia de $115 \mu \mathrm{m}$ entre ambos $(\mathrm{DE}=21$, $\mathrm{n}=2$ ). El pie es incoloro y midió $320 \mu \mathrm{m}$ de longitud en el único ejemplar medido.

Es notable la coincidencia entre los individuos designados aquí a Triphora sp. y los fósiles proveniente de la costa este de Venezuela descritos por Jung (1975, p. 118, Figura 47) como Triphora sp.

Los ejemplares de esta especie fueron escasos, el promedio fue de una larva por mes (Tabla 1).

Caenogastropoda-Neotaenioglossa: Triphorinae (Triphoridae)

La protoconcha larval es espiral cónica, levógira. Los dos primeros individuos registrados midieron 120 y $240 \mu \mathrm{m}$ con alrededor de dos anfractos formados. Individuos completamente desarrollados midieron $510 \mu \mathrm{m}$ de longitud máxima $(\mathrm{DE}=$ $14, \mathrm{n}=2$ ) y estaban formados por cuatro anfractos. La mitad adapical del anfracto del cuerpo es liso en la región ventral y presenta cinco relieves espirales finos en el hombro. La apertura es subcuadrangular (Fig. 6A). No presenta canal sifonal. El resto de los anfractos presentan costillas axiales y dos relieves espirales en la región media del anfracto (Fig. 6B). La protoconcha embrionaria es muy distintiva, es globular y esta ornamentada con líneas espirales formadas por relieves en forma de cruz (gránulos en forma de "T" sensu Marshall 1983). Las coincidencias de las barras verticales otorgan un aspecto cuadriculado a gran parte de la protoconcha (Fig. 6C). El diámetro de la protoconcha embrionaria, medido en un ejemplar fue de $135 \mu \mathrm{m}$.

Las partes blandas (Fig. 6D) se caracterizan como sigue: el velo es bilobulado, despigmentado y asimétrico. El lóbulo derecho es ovalado y mide $475 \mu \mathrm{m}$ de longitud $(\mathrm{DE}=35, \mathrm{n}=2)$, el lóbulo izquierdo es redondeado y mide $200 \mu \mathrm{m}$ de diámetro $(\mathrm{DE}=0, \mathrm{n}=2)$. Los tentáculos miden $75 \mu \mathrm{m}$ de longitud ( $\mathrm{DE}=7, \mathrm{n}=2$ ). Los ojos son negros y se ubican a $150 \mu \mathrm{m}$ de distancia entre sí. El pie es incoloro y mide $230 \mu \mathrm{m}$ de longitud $(\mathrm{DE}=28, \mathrm{n}=2)$.

Las larvas de Triphora sp. y Triphorinae son notoriamente similares en varios caracteres, tales como sentido de la espiralización, ornamentación y tamaño de la concha larval y forma de las partes blandas. La diferenciación se estableció claramente al observar la ornamentación de la protoconcha embrionaria mediante microscopía electrónica de barrido

Esta especie fue muy escasa, al igual que la especie Triphora sp., alcanzó apenas un promedio de una larva por mes (Tabla 1). 
Caenogastropoda-Neotaenioglossa: Janthina sp. (Janthinidae)

La protoconcha larval es espiral cónica, dextrógira, compuesta por 3,5 anfractos, mide $520 \mu \mathrm{m}$ de longitud $(\mathrm{DE}=34,6 ; \mathrm{n}=3)$. La concha es transparente, la columela anaranjada. La apertura es redondeada y de borde engrosado (Fig. 7A), no presenta pico en el borde de la apertura ni canal sifonal. La protoconcha larval presenta todos los anfractos ornamentados por relieves axiales (Fig. 7B). Cada relieve tiene muescas triangulares que le dan aspecto dentado (Fig. 7C). La protoconcha embrionaria posee una franja de estrías perpendiculares a la sutura inicial. El resto de su superficie es lisa (Fig. 7D). La protoconcha embrionaria mide $127 \mu \mathrm{m}$ de diámetro promedio $(\mathrm{DE}=5,6 ; \mathrm{n}=2)$.

Debido al bajo número de ejemplares colectados la medición de las partes blandas (Fig. 7E) se realizó sólo en un ejemplar. El velo es bilobulado, asimétrico, pigmentado de color morado cerca de
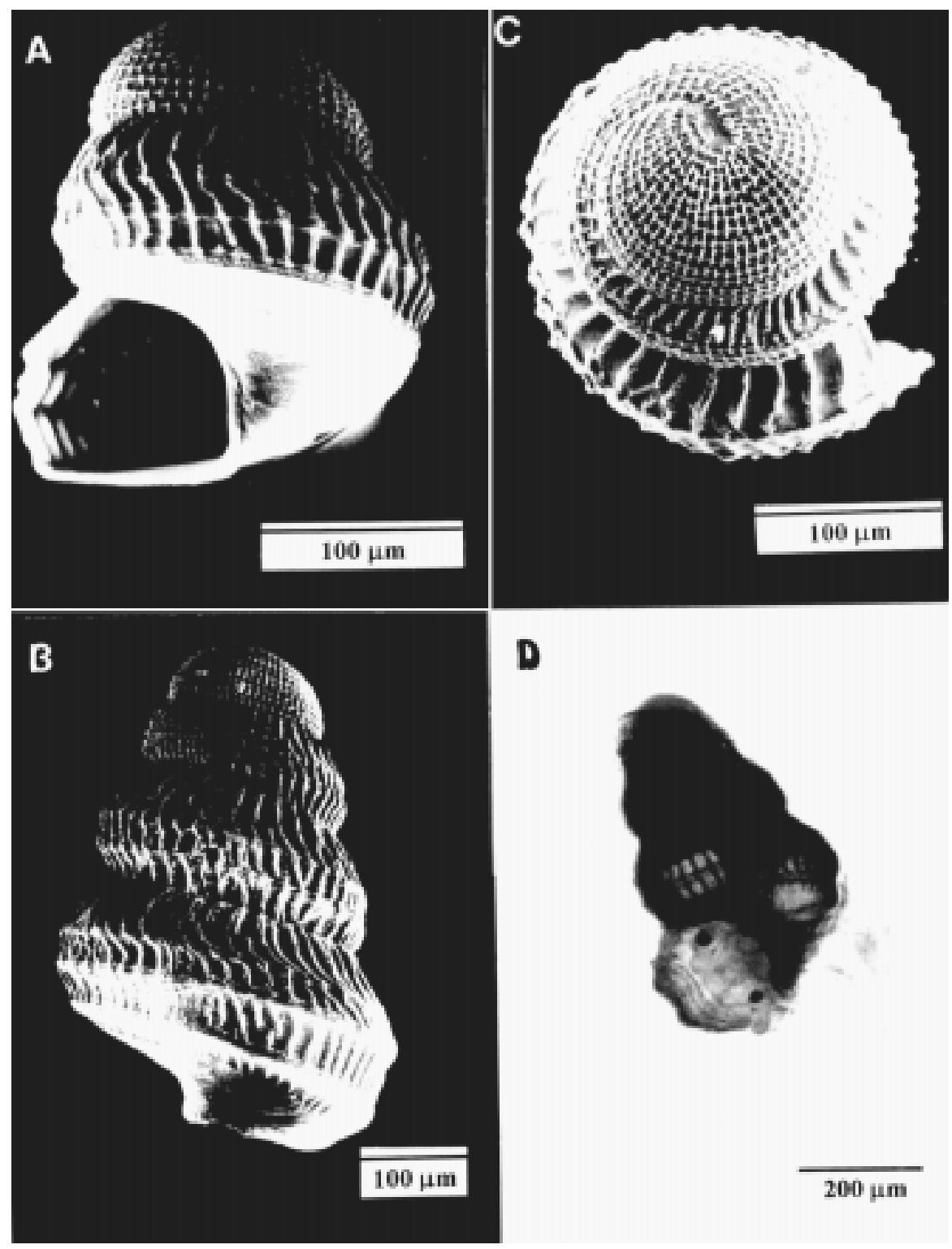

D

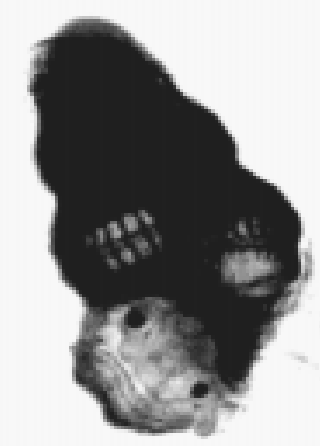

$200 \mu \mathrm{m}$

Fig. 6: Triphoridae: (A) vista ventral de la protoconcha larval (esta protoconcha corresponde a una larva que no ha completado su desarrollo); (B) vista dorsal de la protoconcha larval; (C) protoconcha embrionaria; (D) Larva viva en vista frontal.

Triphoridae: (A) ventral view of larval protoconch (this shell belong to a larva that has not completed development); (B) dorsal view of larval protoconch; (C) embryonic protoconch; (D) frontal view of a living larva. 
la región cefálica. Los lóbulos son redondeados, el derecho mide $450 \mu \mathrm{m}$ de diámetro, el izquierdo, $350 \mu \mathrm{m}$. Los tentáculos miden $110 \mu \mathrm{m}$ de longitud. Los ojos son negros, se ubican a $150 \mu \mathrm{m}$ de distancia uno de otro. El pie es romboidal, despigmentado. En el borde inferior de la boca presenta una mancha negra de $20 \mu \mathrm{m}$ de diámetro.

La forma general de la concha, la ornamentación de la protoconcha embrionaria y larval y particularmente los relieves dentados en esta última, permiten asignar con seguridad estos ejem- plares a la familia Janthinidae, género Janthina (ver Robertson 1971, Lámina V, Figura 17 y 18).

Esta especie fue muy escasa en los muestreos, alcanzó en promedio una larva por mes (Tabla 1).

\section{Caenogastropoda-Neotaenioglossa: Littorinidae}

La protoconcha larval es espiral cónica, dextrógira, de color amarillo, formada por 2,5 a 2,63 anfractos, mide $396 \mu \mathrm{m}$ de longitud máxima

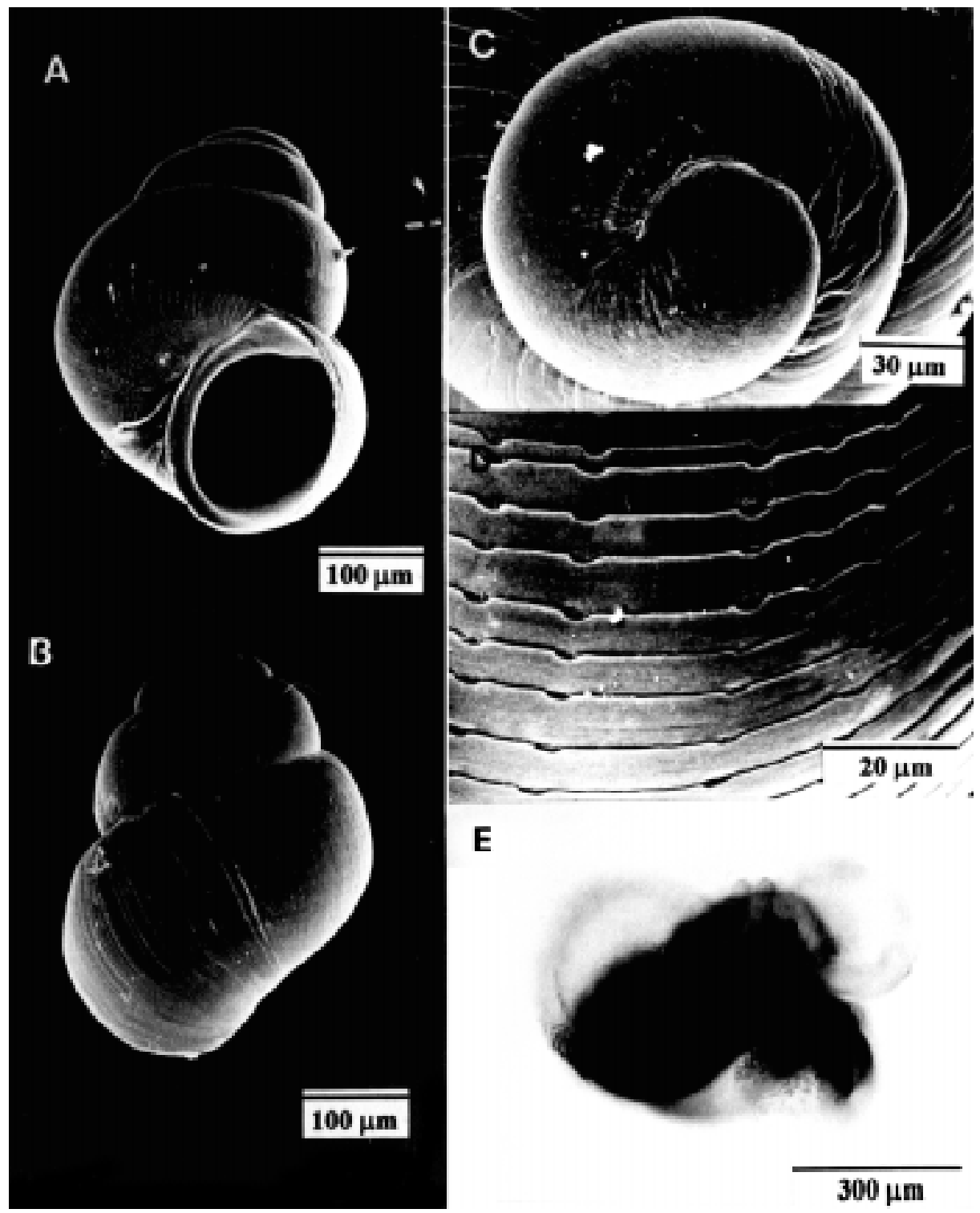

Fig. 7: Janthinidae: (A) vista ventral de la protoconcha larval; (B) vista dorsal de la protoconcha larval; (C) protoconcha embrionaria; (D) detalle de los axiales dentados de la protoconcha; (E) larva viva en vista frontal.

Janthinidae: (A) ventral view of larval protoconch; (B) dorsal view of larval protoconch; (C) embryonic protoconch; (D) detail of the serrated axial of the protoconch; (E) frontal view of a living larva. 
$(\mathrm{DE}=25,8 ; \mathrm{n}=6)$. La apertura es redondeada (Fig. 8A), de borde recto y engrosado (Fig. 8B), no presenta pico ni canal sifonal. El segundo anfracto presenta gránulos esparcidos irregularmente. En los demás anfractos los gránulos se ordenan formando líneas suavemente onduladas (Fig. 8C). En el anfracto del cuerpo, cerca de la sutura y en la periferia lateroventral de la apertura, las líneas son zigzagueantes (Fig. 8B). La protoconcha embrionaria mide $104,3 \mu \mathrm{m}$ de diámetro $(\mathrm{DE}=15,3 ; \mathrm{n}=5)$, presenta una ornamen- tación compuesta de granulaciones finas distribuidas en bandas espirales (Fig. 8D).

Las partes blandas (Fig. 8E) se caracterizan como sigue: el velo es bilobulado, despigmentado y asimétrico. Los lóbulos son semicirculares, el derecho mide $230 \mu \mathrm{m}$ en su parte más ancha (DE $=25,5 ; \mathrm{n}=5)$ y el izquierdo, $178 \mu \mathrm{m}(\mathrm{DE}=19,4$; $\mathrm{n}=5)$. El pie es redondeado, pigmentado negro, mide $294 \mu \mathrm{m}$ de longitud $(\mathrm{DE}=49, \mathrm{n}=5)$. Los ojos son negros, se ubican en el lado de cada tentáculo a una distancia de $144 \mu \mathrm{m}$ entre ambos
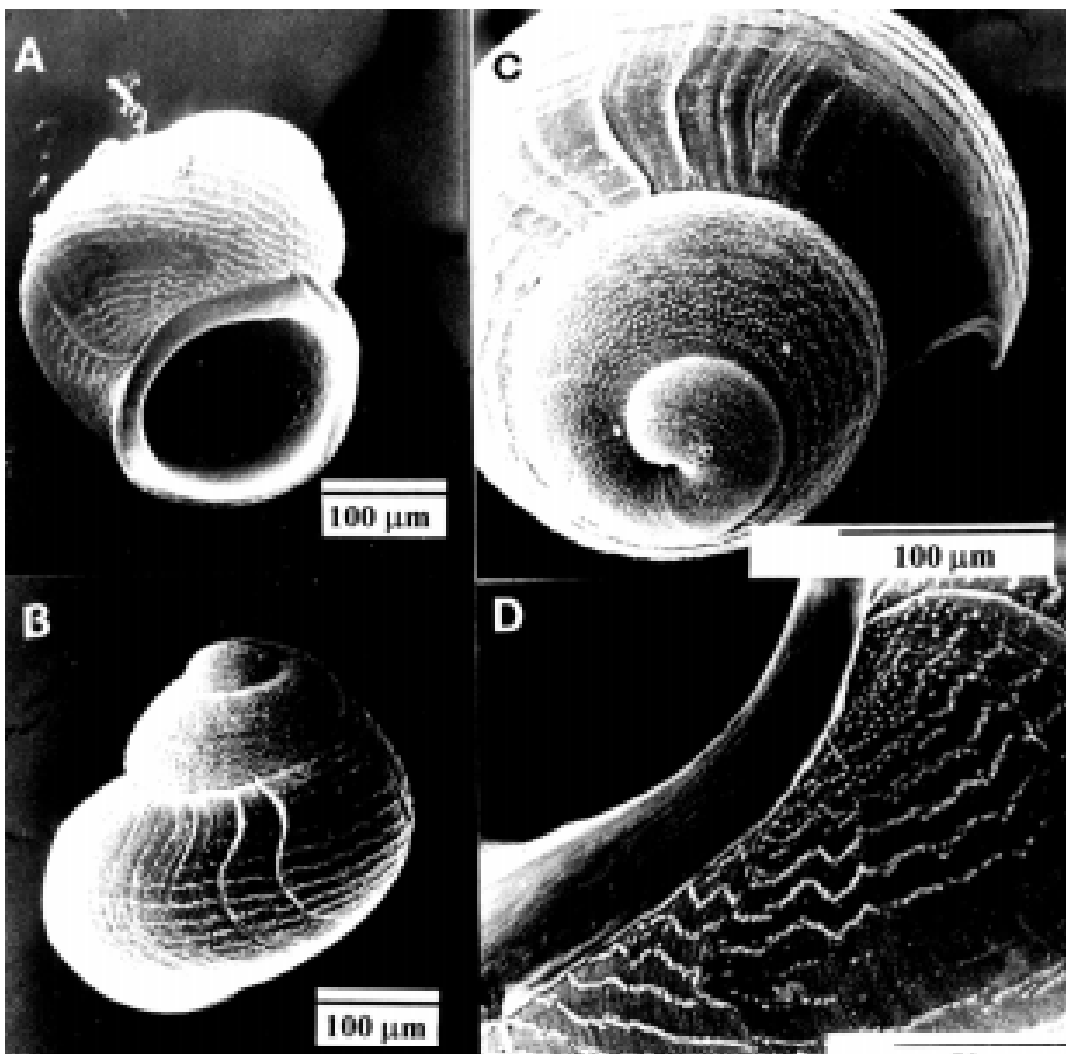

D
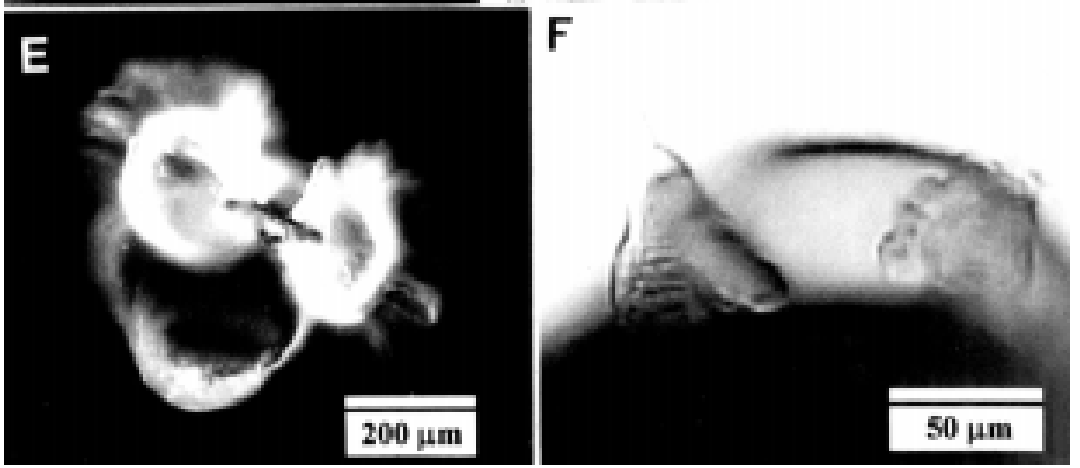

Fig. 8: Littorinidae: (A) vista ventral de la protoconcha larval; (B) vista dorsal de la protoconcha larval; (C) protoconcha embrionaria; (D) detalle de la ornamentación de la concha y del borde engrosado de la apertura; (E) larva viva en vista frontal; (F) detalle de los tentáculos.

Littorinidae: (A) ventral view of larval protoconch; (B) dorsal view of larval protoconch; (C) embryonic protoconch; (D) detail of shell sculpture and wide border of the aperture; (E) frontal view of a living larva; (F) detail of tentacles. 
$(\mathrm{DE}=23,3 ; \mathrm{n}=5)$. Los tentáculos de la veliger de esta especie son muy particulares, miden $118 \mu \mathrm{m}$ de longitud, en vista frontal presentan cuatro proyecciones romas en su parte media (Fig. 8F), vistas desde arriba, las proyecciones asemejan una roseta.

Estas estructuras tentaculares se han observado en estos ejemplares y en larvas de Nodilittorina peruviana (M.S. Romero resultados no publicados). Larvas de N. peruviana cultivadas en laboratorio alcanzan una longitud promedio de 343 $\mu \mathrm{m}(\mathrm{DE}=10,59 ; \mathrm{n}=10)$, presentan una concha larval de forma similar a la especie aquí descrita, pero la ornamentación del anfracto del cuerpo no presenta relieves en zigzag (M.S. Romero resultados no publicados) sino espirales. Estas observaciones permitieron establecer que la especie descrita en este trabajo no corresponde $N$. peruviana sino a una especie relacionada.

Esta especie se presentó en bajo número y en forma esporádica. La mayor abundancia se registró en el mes de diciembre con 6 ejemplares (Tabla 1).

Caenogastropoda-Neogastropoda: Crassilabrum crassilabrum (Sowerby, 1834) (Muricidae)

Concha espiral cónica, globular, dextrógira, de color ámbar, muy transparente. Mide $590 \mu \mathrm{m}$ de longitud máxima ( $\mathrm{DE}=65,6 ; \mathrm{n}=3)$, compuesta por 2,63 anfractos. La apertura es sinusígera, el labio externo de la apertura presenta un pico ancho, redondeado, denticulado y un canal sifonal con ornamentación reticulada diagonal (Fig. 9A). La concha larval es de apariencia lisa con pequeños gránulos en su superficie (Fig. 9B). La protoconcha embrionaria esta ornamentada por gránulos finos, distribuidos uniformemente (Fig. 9C), mide en promedio $205 \mu \mathrm{m}$ de diámetro (DE $=25,8 ; \mathrm{n}=2$ ).

Las partes blandas (Fig. 9D) se caracterizan como sigue: el velo es bilobulado, los lóbulos son asimétricos y tienden a ser tetralobulados. El lóbulo derecho mide $450 \mu \mathrm{m}$ de alto ( $\mathrm{DE}=70,7$; $\mathrm{n}=2)$ por $350 \mu \mathrm{m}$ de ancho $(\mathrm{DE}=0, \mathrm{n}=2)$. El velo carece de pigmentación. Los tentáculos son de forma trapezoide, simétricos, cortos, midieron 50 $\mu \mathrm{m}$ de longitud $(\mathrm{DE}=0, \mathrm{n}=2)$. Los ojos son de color negro, ubicados en posición horizontal cerca de la base de los tentáculos, comparados a otras especies, se encuentran muy separados, 223 $\mu \mathrm{m}$ entre ambos $(\mathrm{DE}=25, \mathrm{n}=3)$. El pie presenta una forma distintiva (Fig. 9E). En estados tempranos de desarrollo, el pie tiene forma de pentágono y los vértices que forman el metapodio son espatulados. El pie de larvas competentes es ámbar cristalino, presenta lóbulos mesopodiales asimétricos. La longitud sólo se registró en dos larvas y fue de 250 y $300 \mu \mathrm{m}$. La mitad proximal de la suela del pie es pigmentada de color negro, la pigmentación es más concentrada hacia los bordes laterales y en el centro (Fig. 9E. Las larvas de esta especie, tanto las capturadas en el plancton como las cultivadas en laboratorio tienden a nadar con el dorso hacia arriba y la región ventral cerca del fondo de la cápsula Petri.

Esta especie fue identificada como Crassilabrum crassilabrum (Sowerby, 1834), Muricidae. La identificación se realizó comparando los ejemplares recolectados en el plancton con larvas cultivadas en laboratorio. Las ovicápsulas utilizadas para obtener larvas cultivadas fueron recolectadas durante el proceso de ovipostura de los adultos lo cual asegura la identidad de las larvas. Además, las cápsulas utilizadas concuerdan a la descripción realizada por Cañete (1992).

Crassilabrum crassilabrum fue poco frecuente o estuvo ausente los tres primeros meses de muestreo. En el muestreo del 30 de mayo alcanzó un $71 \%$ de la muestra (Tabla 1).

\section{Caenogastropoda-Neogastropoda: Xanthochorus cassidiformis (Blainville, 1832) Muricidae}

Concha espiral cónica, dextrógira, de color ámbar claro, compuesta de 1,88 anfractos, mide $760 \mu \mathrm{m}$ de longitud máxima promedio $(\mathrm{DE}=40 ; \mathrm{n}=5)$. El borde externo de la apertura es recto y presenta canal sifonal (Fig. 10A). La concha larval presenta gránulos más grandes y distanciados que la protoconcha embrionaria (Fig. 10B). La protoconcha embrionaria esta ornamentada por gránulos finos, distribuidos uniformemente por la superficie (Fig. 10C). El diámetro de la protoconcha embrionaria medido en un ejemplar fue de $333 \mu \mathrm{m}$.

Las partes blandas (Fig. 10D) se caracterizan como sigue: el velo tiende a ser tetralobulado, mide $600 \mu \mathrm{m}$ de alto $(\mathrm{DE}=70,7 ; \mathrm{n}=2)$ y $300 \mathrm{de}$ ancho (una sola medición), presenta pigmentación morada en los cuatro extremos distales. Los tentáculos son simétricos, miden $70 \mu \mathrm{m}(\mathrm{DE}=$ 15,$8 ; \mathrm{n}=5$ ). Los ojos son negros, se ubican en la base de los tentáculos, con una distancia de $80 \mu \mathrm{m}$ entre ambos $(\mathrm{DE}=25,5 ; \mathrm{n}=5)$. El pie presenta pigmento café en la parte media, mide $602 \mu \mathrm{m}$ de longitud ( $\mathrm{DE}=116,7 ; \mathrm{n}=5$ ) (Fig. 10E).

La comparación de larvas recolectadas en el plancton con larvas cultivadas en laboratorio de parentales conocidos, permitió identificar esta especie como Xanthochorus cassidiformis 
(Blainville, 1832) Muricidae. También se consideraron las observaciones y descripciones dadas por Gallardo \& González (1984) sobre esta misma especie.

Esta especie fue muy poco frecuente y esporádica en los muestreos. Se capturó en marzo, abril y diciembre de uno a cinco ejemplares (Tabla 1).
Caenogastropoda-Neogastropoda: Salitra radwini Marincovich, 1973 (Collumbellidae)

La concha larval es espiral cónica, dextrógira, de color café-rojizo, mide $1.125 \mu \mathrm{m}$ de longitud máxima, formada por 4,63 anfractos. La apertura es sinusígera, el labio externo presenta un pico

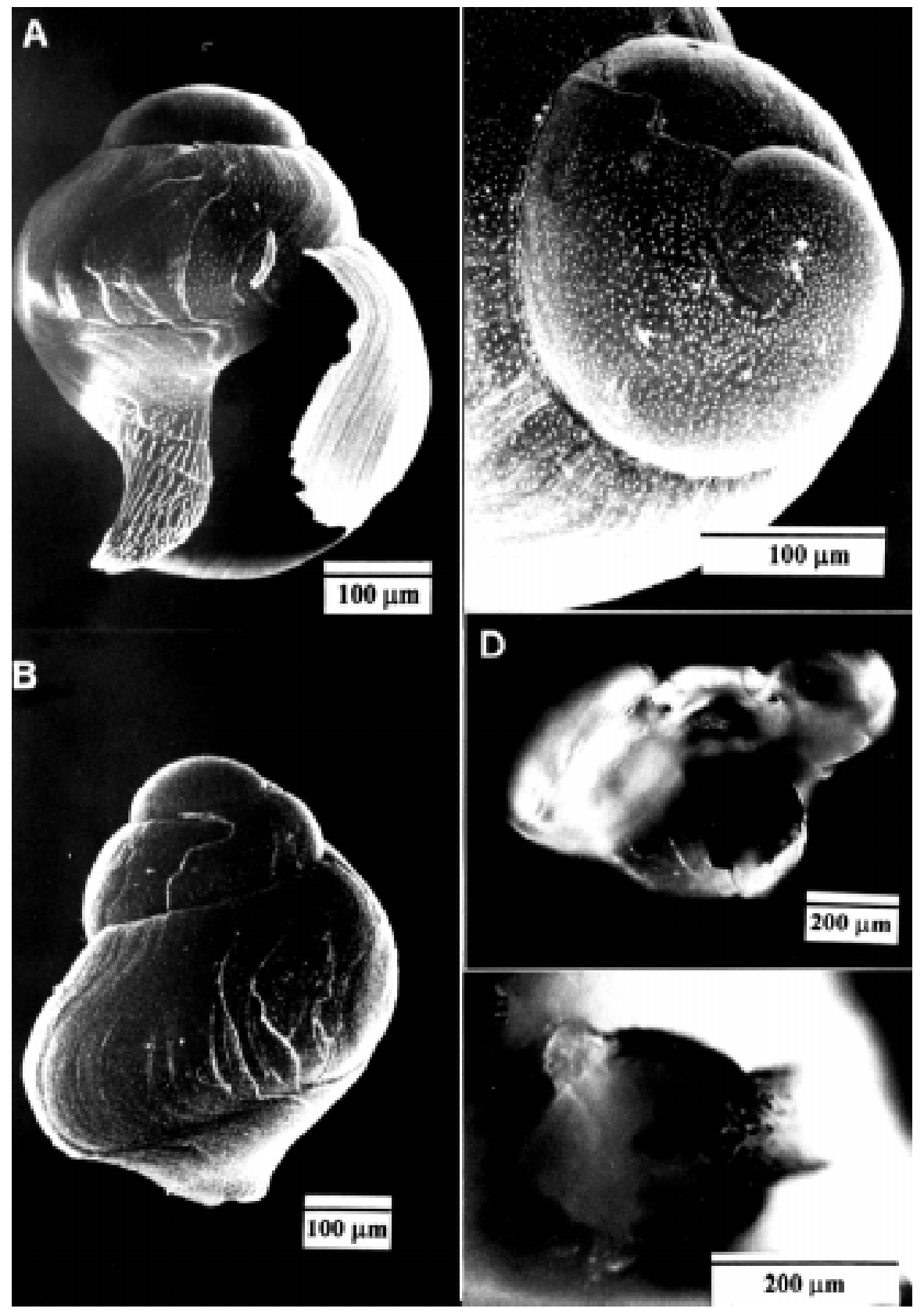

Fig. 9: Crassilabrum crassilabrum (Sowerby, 1834), Muricidae: (A) vista ventral de la protoconcha larval; (B) vista dorsal de la protoconcha larval; (C) protoconcha embrionaria; (D) larva viva en vista frontal; (E) detalle de la pigmentación del pie.

Crassilabrum crassilabrum (Sowerby, 1834), Muricidae: (A) ventral view of larval protoconch; (B) dorsal view of larval protoconch; (C) embryonic protoconch; (D) frontal view of a living larva; (E) detail of pigmentation of foot. 
ancho y redondeado (Fig. 11 A). A ambos lados de éste, el labio externo se encuentra curvado hacia el exterior (Fig. 11B). La protoconcha larval presenta una ornamentación característica formada por relieves dispuestos en forma oblicua respecto al eje axial, formando un reticulado diagonal. La región media del anfracto del cuerpo presenta dos relieves espirales. Entre los relieves espirales y en la región abapical del anfracto del cuerpo, la ornamentación pierde el ordenamiento diagonal y tiende a ser un reticulado irregular (Fig. 11B y
11C). La protoconcha embrionaria presenta una ornamentación muy particular compuesta por relieves espirales zigzagueantes, discontinuos y estrechamente cercanos entre ellos (Fig. 11D). La protoconcha embrionaria midió $181 \mu \mathrm{m}$ de diámetro ( $\mathrm{DE}=7,3 ; \mathrm{n}=5)$.

Las partes blandas se han observado en dos larvas y medido en una larva que completó su desarrollo en laboratorio 45 días después de su recolección (Fig. 11E). El velo es tetralobulado con lóbulos asimétricos. Los lóbulos posteriores

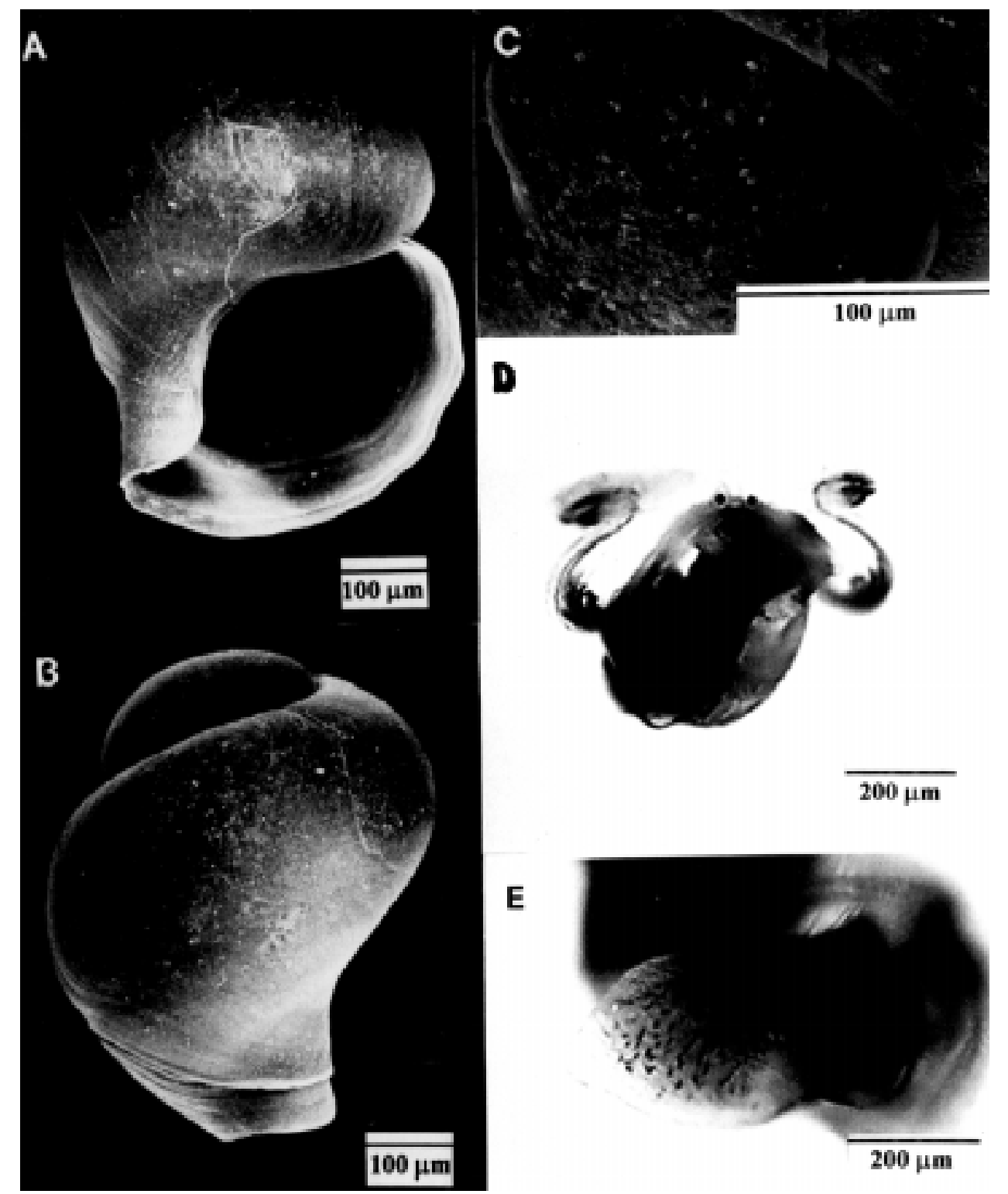

Fig. 10: Xanthochorus cassidiformis (Blainville, 1832), Muricidae: (A) vista ventral de la protoconcha larval; (B) vista dorsal de la protoconcha larval; (C) protoconcha embrionaria; (D) larva viva en vista frontal; (E) detalle de la pigmentación punteada del pie.

Xanthochorus cassidiformis (Blainville, 1832), Muricidae: (A) ventral view of larval protoconch; (B) dorsal view of larval protoconch; (C) embryonic protoconch; (D) frontal view of a living larva; (E) detail of dotted pigmentation of foot. 
están orientados hacia la parte anterior. El lóbulo posterior izquierdo mide $1.875 \mu \mathrm{m}$ de longitud y el derecho $1.625 \mu \mathrm{m}$. Los lóbulos anteriores son ovalados, miden $1.375 \mu \mathrm{m}$ de longitud el derecho y $1.250 \mu \mathrm{m}$ el izquierdo. En estados tempranos de desarrollo cuando el velo es aún bilobulado, los lóbulos presentan manchas de color morado cerca de la región cefálica. Los tentáculos son simétricos, filiformes, miden $100 \mu \mathrm{m}$ de longitud. Los ojos se ubican en posición horizontal en la base de los tentáculos a una distancia de $160 \mu \mathrm{m}$ entre

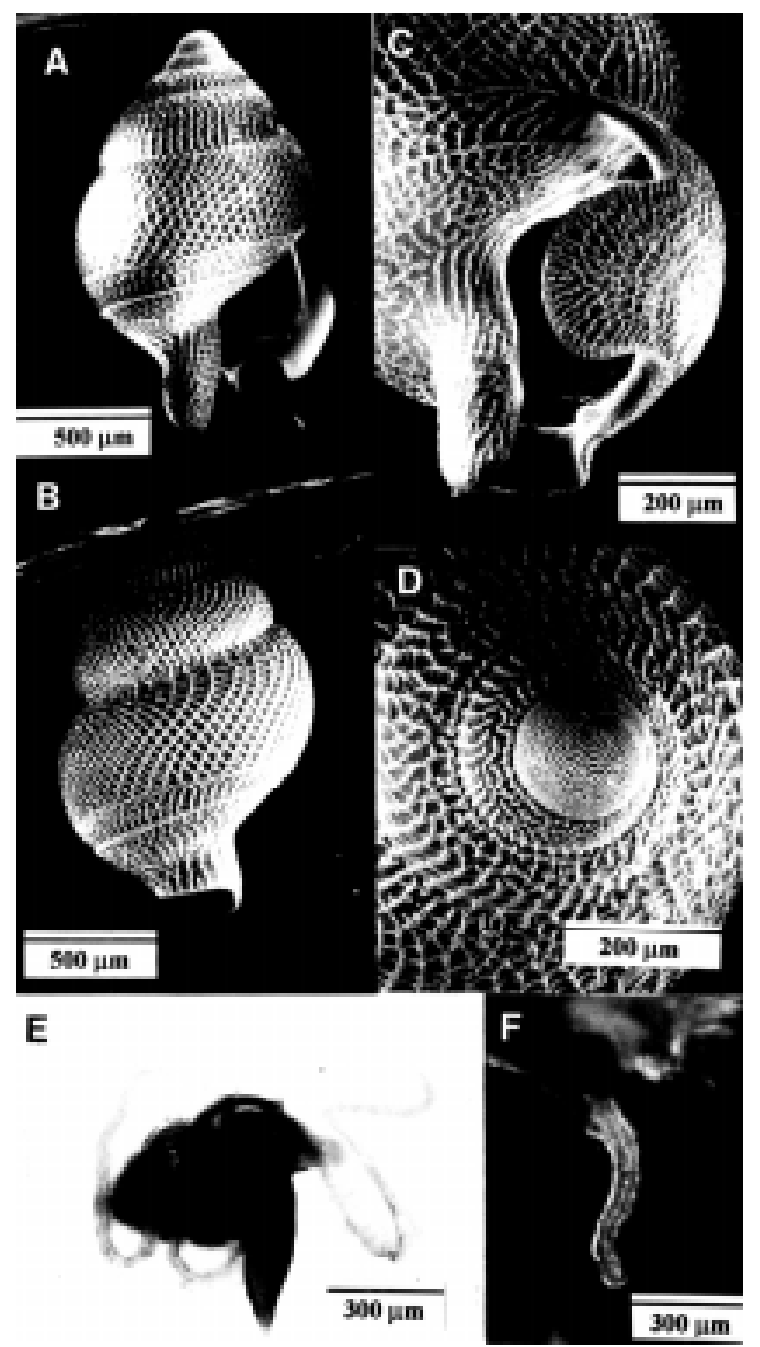

Fig. 11: Salitra radwini Marincovich, 1973,

Collumbellidae: (A) vista ventral de la protoconcha larval; (B) vista dorsal de la protoconcha larval; (C) detalle de la apertura; (D)protoconcha embrionaria; (E) larva viva en vista frontal; (F) detalle de la proyección metapodial.

Salitra radwini Marincovich, 1973, Collumbellidae: (A) ventral view of larval protoconch; (B) dorsal view of larval protoconch; (C) detail of aperture; (D) embryonic protoconch; (E) frontal view of a living larva; (F) detail of metapodial projection. ambos. El pie mide $850 \mu \mathrm{m}$ de longitud total, tiene forma triangular y el metapodio está representado por una larga proyección en forma de dedo que mide $300 \mu \mathrm{m}$ de longitud (Fig. 11F). Todo el margen del pie incluyendo la proyección es transparente. El centro de la suela del pie es de color café con manchas negras.

Esta especie es muy frecuente en comparación a las otras. Se presentó en la mayor parte de los muestreos aunque, generalmente, en estados tempranos post-eclosión.

Esta especie se adjudicó a la especie Salitra radwini Marincovich, 1973, familia Collumbellidae, sobre la base de la descripción de la protoconcha que realizó Marincovich (1973) y a la esquematización y redescripción realizadas por Guzmán et al. (1998).

Cabe hacer notar la similitud de Salitra radwini y Mitrella sp. en estados tempranos de desarrollo. Coinciden en la forma y pigmentación del velo, la forma del pie con la proyección digitiforme en el metapodio y la forma general de la concha. En estado competente ambas pierden la pigmentación velar y desarrollan cuatro lóbulos aunque de forma diferente.

Caenogastropoda-Neogastropoda: Mitrella $s p$. (Collumbellidae)

La concha larval es espiral cónica, dextrógira y de color amarillo-ámbar, formada por 3,5 anfractos y mide $1.110 \mu \mathrm{m}$ de longitud máxima $(\mathrm{DE}=14, \mathrm{n}$ $=2$ ). La apertura es sinusígera, el borde externo de la apertura presenta un pico ancho y redondeado, posee un canal sifonal con ornamentación reticulada (Fig. 12A). La concha larval esta ornamentada por gránulos dispersos. En la región dorsal del anfracto del cuerpo la superficie es lisa (Fig. 12B). La protoconcha embrionaria esta ornamentada con gránulos finos distribuidos uniformemente (Fig. 12C). El diámetro de la protoconcha embrionaria medido en un ejemplar fue de $99,2 \mu \mathrm{m}$.

Las partes blandas (Fig. 12D) se caracterizan como sigue: el velo es tetralobulado, los lóbulos son simétricos redondeados de $750 \mu \mathrm{m}$ de diámetro $(\mathrm{DE}=70, \mathrm{n}=2)$. En estados tempranos de desarrollo cuando, el velo es aún bilobulado, los lóbulos presentan manchas de color morado próximo a la región cefálica. Los tentáculos son simétricos, filiformes, miden $250 \mu \mathrm{m}$ de longitud. Los ojos son negros y se ubican al lado de los tentáculos a una distancia de $250 \mu \mathrm{m}$ entre ambos $(\mathrm{DE}=$ 70,$7 ; \mathrm{n}=2$ ). El pie es amarillo claro en los bordes y pigmentado gris oscuro en el centro. La longitud del pie relajado medido en una larva compe- 
tente fue de $750 \mu \mathrm{m}$ de largo. El pie presenta lóbulos mesopodiales asimétricos y una proyección metapodial digitiforme (Fig. 12E).

Estos ejemplares muestran gran similitud con la especie descrita por Thiriot-Quiévreux (1980), Mitrella lunata (p. 7, Figuras 49-52), coincidiendo en todos los caracteres generales de forma y ornamentación de la concha larval. Cabe destacar la coincidencia en la forma curvada del canal sifonal, el que presenta relieves nudosos longitudinales que nacen desde la columela y que se encuentran atravesados por relieves perpendiculares en el extremo distal. Esta especie fue asignada a la familia Collumbellidae, género Mitrella.

Esta especie fue poco frecuente y esporádica, recolectándose sólo en las muestras del 1 de marzo y 30 de mayo (Tabla 1).

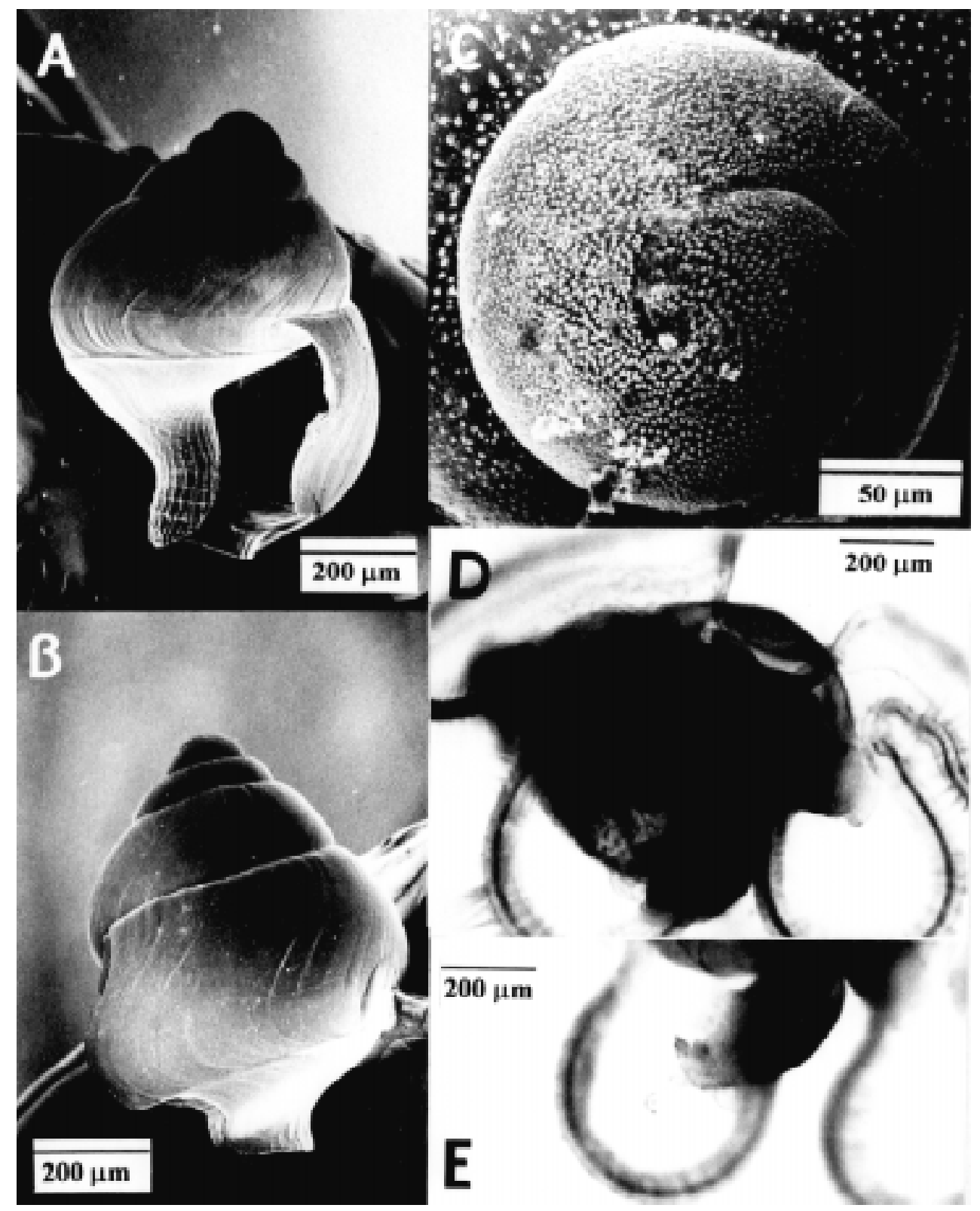

Fig. 12: Mitrella sp., Collumbellidae: (A) vista ventral de la protoconcha larval; (B) vista dorsal de la protoconcha larval; (C) protoconcha embrionaria; (D) larva viva en vista frontal; (E) detalle de la proyección metapodial

Mitrella sp., Collumbellidae: (A) ventral view of larval protoconch; (B) dorsal view of larval protoconch; (C) embryonic protoconch; (D) frontal view of a living larva; (E) detail of metapodial projection. 
Caenogastropoda-Neogastropoda: Nassarius dentifer (Powys, 1835) (Nassaridae)

La protoconcha larval es espiral cónica, dextrógira, de color café, formada por 3,25 anfractos, mide $1.081 \mu \mathrm{m}$ de longitud máxima $(\mathrm{DE}=285,6 ; \mathrm{n}=14)$. El borde externo de la apertura es recto y presenta canal sifonal. En vista dorsal la espira del cuerpo presenta nueve estrías espirales (Fig. 13A). La protoconcha embrionaria presenta una ornamentación compuesta de graduaciones distribuidas uniformemente por la superficie (Fig. 13B) y mide $163 \mu \mathrm{m}$ de diámetro $(\mathrm{DE}=17,7 ; \mathrm{n}=4)$.

Las partes blandas (Fig. 13C) se caracterizan como sigue: el velo es tetralobulado con lóbulos simétricos de $1.300 \mu \mathrm{m}$ de longitud $(\mathrm{DE}=635 ; \mathrm{n}$ $=6$ ). El velo presenta una pigmentación característica compuesta por una mancha púrpura muy obscura ubicada en la región centro-distal del lóbulo, abarcando 0,5 a 0,25 de éste. Los tentáculos son simétricos, miden $216 \mu \mathrm{m}$ de longitud (DE $=85,3 ; \mathrm{n}=5$ ). Cerca de la base, los tentáculos son engrosados y en esa región, en posición lateral se encuentran los ojos de color negro. El pie presenta procesos tentaculares en el extremo del propodio. El extremo del metapodio se encuentra dividido por una muesca. La suela del pie esta pigmentada por puntos café, distribuidos homogéneamente. En el centro de la suela, la pigmentación se concentra formando una figura con forma de " $T$ " donde la barra horizontal se ubica en el borde del propodio y la barra vertical alcanza un poco más de la mitad del pie. Bajo ésta y en forma continuada se encuentra una mancha de pigmento de forma ovalada. El pie mide $767 \mu \mathrm{m}$ de longitud $(\mathrm{DE}=181,7 ; \mathrm{n}=10)$.

Seis ejemplares de la esta especie metamorfosearon en laboratorio. Uno de ellos alcanzó una longitud de $0,8 \mathrm{~mm}$ a los tres meses de edad. Este ejemplar fue identificado como Nassarius dentifer (Powys, 1835), Nassaridae.

Caenogastropoda-Neogastropoda: Nassarius sp. (Nassaridae)

La protoconcha larval es espiral cónica, dextrógira, de color amarillo, formada por 3,25 anfractos, mide $1.491 \mu \mathrm{m}$ de longitud máxima $(\mathrm{DE}=214,8 ; \mathrm{n}=14)$. El borde externo de la apertura es recto y presenta canal sifonal. La espira del cuerpo presenta ornamentación compuesta sólo por seis relieves espirales en la mitad adapical (Fig. 14A). La protoconcha embrionaria presenta una ornamentación compuesta de granulaciones finas distribuidas uniformemente
(Fig. 14B) y mide $168 \mu \mathrm{m}$ de diámetro ( $\mathrm{DE}=18$, $\mathrm{n}=3$ ).

Las partes blandas (Fig. 14C) se caracterizan como sigue: el velo es tetralobulado con lóbulos simétricos que miden $2.128 \mu \mathrm{m}$ de longitud $(\mathrm{DE}=$ $535, \mathrm{n}=8$ ). Los lóbulos presentan pigmentación compuesta de puntos morados ubicados en la región centro-distal, abarcando 0,5 a 0,8 de cada lóbulo. Los tentáculos son simétricos, engrosados en la base y en esa porción de los tentáculos, en posición lateral, se ubican los ojos de color

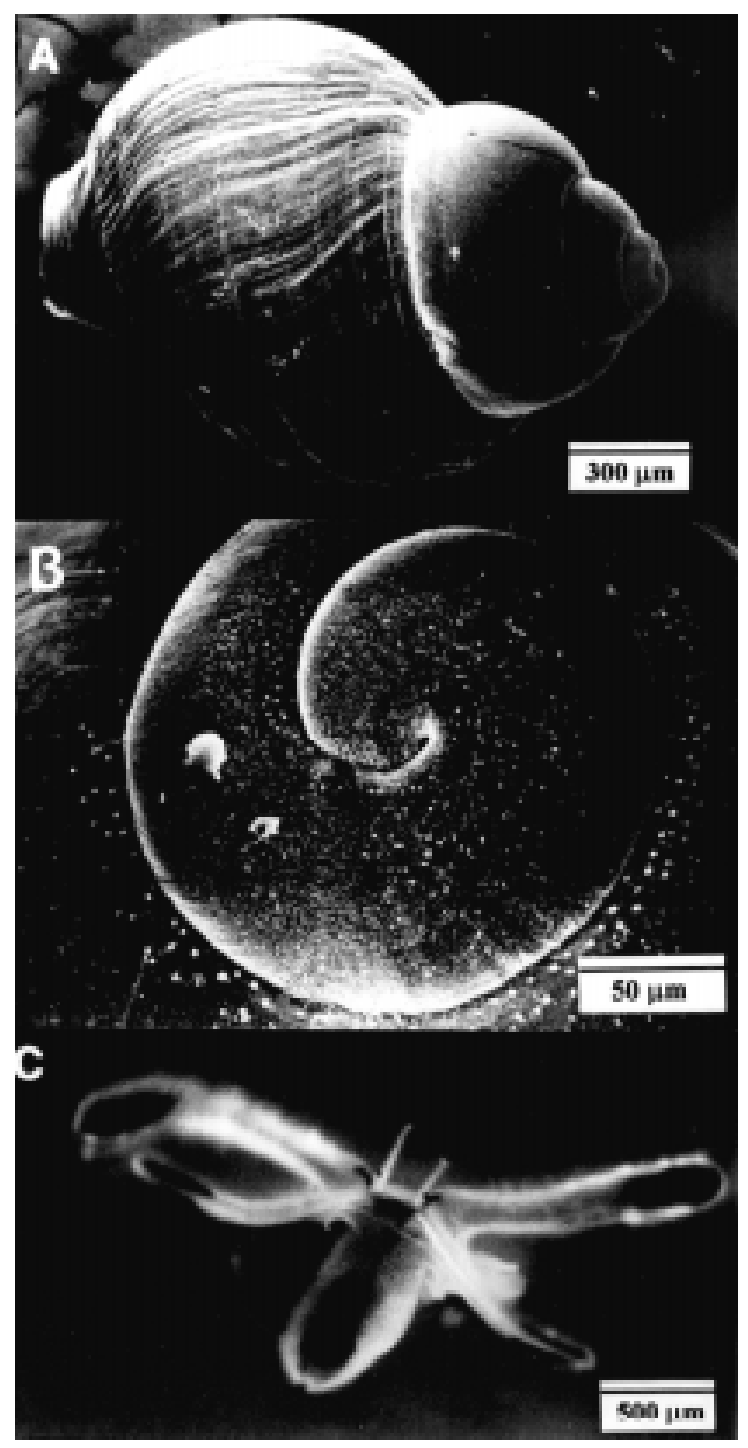

Fig. 13: Nassarius dentifer (Powys, 1835),

Nassaridae: (A) vista dorsal de la protoconcha larval; (B) protoconcha embrionaria; (C) larva viva en vista frontal.

Nassarius dentifer (Powys, 1835), Nassaridae: (A) dorsal view of larval protoconch; (B) embryonic protoconch; (C) frontal view of a living larva. 
negro. El pie es de color blanco en los bordes y la suela del pie esta pigmentada negro en todo su centro. El pie presenta procesos tentaculares laterales en el extremo del propodio.

Las especies Nassarius dentifer y Nassarius sp. son extremadamente similares en morfología, tamaño y proporciones de la concha y partes blandas. Las características que permiten separar las larvas son: en larvas vivas, la pigmentación de los lóbulos velares y del pie. En conchas preparadas para MEB, la escultura espiral en el anfracto del cuerpo.

Esta especie fue asignada a la familia Nassaridae, género Nassarius.

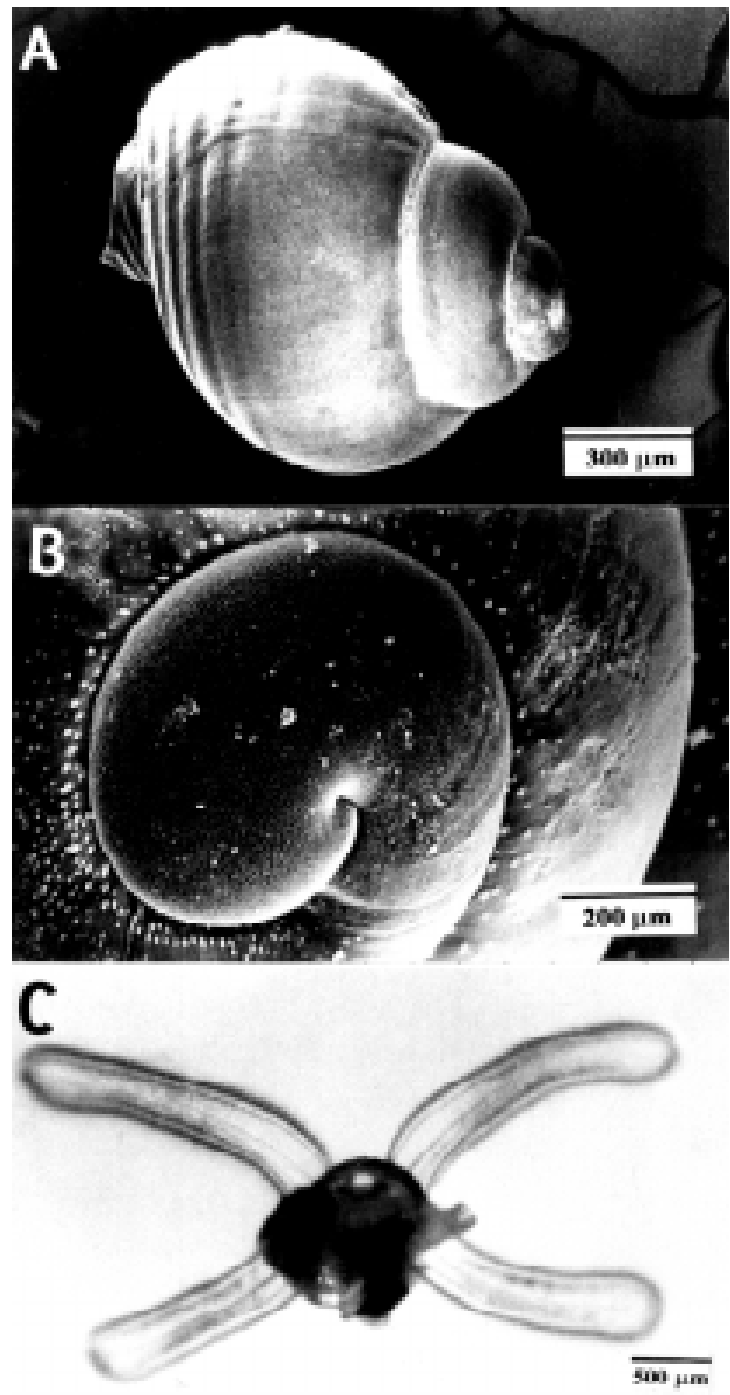

Fig. 14: Nassarius sp., Nassaridae: (A) vista dorsal de la protoconcha larval; (B) protoconcha embrionaria; (C) larva viva en vista frontal.

Nassarius sp., Nassaridae: (A) dorsal view of larval protoconch; (B) embryonic protoconch; (C) frontal view of a living larva.

\section{DISCUSIÓN}

\section{Turritellidae}

Verificar la ocurrencia de larvas en el plancton permite establecer un patrón de distribución temporal que indirectamente da cuenta del ciclo reproductivo de la especie. Además, permite relacionar la presencia de larvas con la época de postura y de ese modo inferir el tiempo de desarrollo en el plancton. Observaciones realizadas durante cinco años en Bahía La Herradura de Guayacán, han permitido establecer eventos reproductivos de Turritella cingulata en primavera y verano (Romero et al. en prensa). Es altamente probable que las larvas capturadas en el muestreo del 1 de marzo correspondan al desove observado en noviembre de 1999. Esto permite inferir una duración planctónica de 4 meses en período de verano. La caracterización de las larvas de $T$. cingulata entregada en este trabajo y los muestreos planctónicos proyectados para los siguientes años permitirán verificar esta estimación.

Características de conchas larvales de Turritella spp. provenientes de muestras fósiles han sido consideradas como caracteres taxonómicos importantes para separar especies y establecer relaciones con otros géneros (Marwick 1957, Allmon 1996, Kowalke 1998b).

Entre las especies recientes que se han descrito se cuenta Turritella variegata presente en el Caribe (Bandel 1976), T. gonostoma del Golfo de California (Allmon et al. 1992) y T. communis presente en el Atlántico (Lebour 1933c, 1937, Thorson 1946, Thiriot-Quiévreux 1969). A pesar de las limitaciones que imponen algunas descripciones parciales, es posible concluir que la morfología Turritella spp., tanto de fósiles como recientes, es simple, conservativa y concuerda con las características observadas en $T$. cingulata. La morfología larval del género Turritella se resume como concha globular, compuesta por 3,5 anfractos (excepto Turritella sp. 2 con 5,5 anfractos), de superficie lisa o con escasa ornamentación, protoconcha embrionaria pequeña entre 100 a 229 um y, aunque algunos autores no lo mencionan, el borde de la apertura es suavemente festoneado (Lebour 1933c, Thorson 1946, Thiriot-Quiévreux 1969, Bandel 1976, Kennedy \& Keegan 1992, Allmon 1996, Kowalke 1998b).

\section{Cerithiidae, Cerithiopsidae}

Las larvas veliger de las familias Cerithiidae y Cerithiopsidae son coincidentes en muchos carac- 
teres tales como: velo bilobulado, asimétrico, despigmentado, concha con varios anfractos, suturas poco marcadas y talla de asentamiento cercana a los $600 \mu \mathrm{m}$ (Thiriot-Quiévreux 1969, 1980, 1982, 1983, Jung 1975, Rodríguez \& ThiriotQuiévreux 1975, Taylor 1975, Marshall 1978, Kowalke 1998b). Otros rasgos como pico rectangular, ensanchado en el extremo anterior y canal sifonal corto, están presentes en todas las especies de Cerithiopsidae y también en algunos Cerithiidae (Lebour 1937, 1945, Thiriot-Quiévreux 1969, 1974, 1980, 1982, Taylor 1975). Este carácter no se observó en las larvas recolectadas en este trabajo debido a lo cual no se asignó a Cerithiopsidae. Debido a lo expuesto, la asignación a familia Cerithiidae queda sujeta a confirmación.

Mayor certeza se ha tenido al asignar una de las especies a Cerithiopsis sp. El género reúne característica tales como concha con cuatro a cinco anfractos suavemente convexos, apertura sinusígera, protoconcha embrionaria con ornamentación granulosa. En algunas especies hay cordones cortos granulosos en la región subsutural y el resto de la superficie es lisa u ornamentada con axiales granulosos opisto o prosoclinos (Lebour 1933c, 1937, Thiriot-Quiévreux 1969, 1974, 1980, Jung 1975, Thiriot-Quiévreux \& Rodríguez 1975, Taylor 1975, Marshall 1978, Nützel 1998). Marshall (1978) realizó una revisión taxonómica extensa de la familia Cerithiopsidae con ejemplares fósiles y recientes de Nueva Zelandia y provenientes de diversos museos del mundo. Esta revisión incluyó la descripción de especies nuevas como Cerithiopsis powelli. La descripción de la protoconcha larval de $C$. powelli coincide en todos los caracteres mencionados con las larvas Cerithiopsis sp. capturadas en este trabajo a excepción de «prominent, sharp, median peripheral carina" en el último anfracto indicado por Marshall (1978, ver Figura 13, p. 109).

Sobre la distribución de Cerithiopsidae en Chile, se cuenta la descripción morfológica que hace Marincovich (1973) de un Cerithiopsis sp. y su presunción sobre la presencia de otras especies entre los especímenes que recolectó en la zona de Iquique. Un aporte más extenso lo realizó Ramírez (1987), quien indicó la presencia de siete especies de Cerithiopsidae distribuidos en Isla de Pascua, Isla Seymour, Estrecho de Magallanes y la Antártida, entre las cuales se cuenta Cerithiopsis powelli Marshall, 1978 en Hotu Iti, Isla de Pascua. En base a las evidencias mencionadas se propone la especie Cerithiopsis powelli como la identificación más probable de los ejemplares Cerithiopsis sp. recolectados en este trabajo en Punta de Lobos.
La presencia de larvas Cerithiidae (?) y Cerithiopsis sp. con desarrollo incompleto en los muestreos que se realizaron en este trabajo, sugieren claramente la presencia de representantes adultos de Cerithiidae y/o Cerithiopsidae en la Cuarta Región de Chile que aún no se han detectado.

\section{Triphoridae}

Marshall (1983), realizó un extenso trabajo sobre identificación específica de miembros de la familia Triphoridae provenientes de varias localidades del Indo-Pacífico. El autor concluyó que existen al menos 1.000 especies y probablemente más que no han sido descritas. En Chile están descritas al menos siete especies de Triphoridae que presentan espiralización levógira (Marincovich 1973, Ramírez 1987). Este carácter es poco frecuente, presentándose en menos del $4 \%$ de los mesogastrópodos chilenos revisados por Ramírez (1987). Contrario a lo esperado, considerando la presencia de ejemplares en estados tempranos de desarrollo, no se encontró registro de especies levógiras para Chile continental. Seis especies están citadas para Isla de Pascua y una para el Archipiélago de Juan Fernández (Ramírez 1987). La ocurrencia de estas larvas en el sitio de recolecta indica la presencia no registrada de especies de la familia Triphoridae en la Cuarta Región.

La morfología de la concha larval de la mayoría de los representantes de Triphoridae es muy característica y deja poco lugar a dudas sobre su identificación (Lebour 1937, 1945, Marshall 1983, Bandel et al. 1997). Además de ser levógira, la concha larval es turriculada, los anfractos presentan gruesas costillas axiales o colabrales atravesadas por dos a tres quillas cordones espirales (Lebour 1933c, 1937, Thiriot-Quiévreux 1969, Taylor 1975, Thiriot-Quiévreux \& Rodríguez 1975, Thiriot-Quiévreux \& Scheltema 1982, Marshall 1983, Bandel 1997). Esta ornamentación que Taylor (1975) describió para $T$. cingulifera, T. perfecta, T. pallida fue denominada Patrón 2 por la autora y concuerda con las especies aquí descritas. Igualmente, el rango de tamaño de la protoconcha, 560-580 $\mu \mathrm{m}$, forma y número de lóbulos del velo y forma del pie de las especies mencionadas y de Triphora perversa son similares a las especies Triphoridae recolectadas en este trabajo (Lebour 1933c, 1937, Fretter \& Graham 1962, Thiriot-Quiévreux \& Scheltema 1982, Bandel 1997, Hickman 1999).

Sobre la protoconcha embrionaria, Marshall (1983) describe los siguientes patrones de ornamentación: gránulos hemiesféricos o en forma de " $\mathrm{T}$ ", líneas espirales en zigzag o reticulado espi- 
ral y cordones axiales. La especie adjudicada a la Subfamilia Triphorinae corresponde al patrón de gránulos en forma de " $T$ " indicando una relación más cercana con las especies denominadas por Marshall (1983) como "el grupo Inella". Este grupo incluye al menos ocho géneros entre los cuales dos especies, Monophorus australica n. sp. Marshall, 1975 y Subulophora rutilans (Hervier, 1897) presentan esta ornamentación característica. Basándose en lo expuesto se asignó los ejemplares colectados en este trabajo a la subfamilia Triphorinae.

\section{Janthinidae}

El hallazgo de la Janthina sp. en estados tempranos de desarrollo durante el muestreo del 30 de mayo sugiere la presencia de reproductores de Janthinidae relativamente cercanos a la zona de estudio. Las especies del género Janthina se caracterizan por sus hábitos pelágicos por lo cual es difícil determinar la extensión de su distribución geográfica. Tres especies se han descrito para diferentes regiones de Chile, dos de ellas específicamente para la zona de Isla de Pascua (Ramírez 1987). La especie J. janthina, cuya ocurrencia se cita para Isla de Pascua, ha sido descrita detalladamente por Robertson (1971) y no concuerda con los caracteres observados en los ejemplares recolectados en este estudio. Ramírez (1987) menciona la ocurrencia de ejemplares de J. globosa en la playa después de fuertes marejadas en la localidad de Papudo, Quinta Región, pero las larvas de esta especie descritas por Taylor (1975), concuerdan sólo parcialmente con las observadas en este trabajo. Las diferencias radican principalmente en la carencia de pigmento en el velo y secundariamente en la coloración de la concha, opérculo y pie.

\section{Littorinidae}

La estructura en forma de roseta que poseen los tentáculos de esta especie la diferencia de todas las restantes especies capturadas en el presente estudio. No se encontró ningún otro ejemplo similar en la literatura revisada y se desconoce si esta estructura presenta una función particular. Cabe destacar que esta especie, al igual que Janthina sp. permanecen flotando "insistentemente" en la superficie aún cuando el agua es agitada. La singular estructura de los tentáculos podría tener alguna relación con la boyantez observada en estas larvas. Se asignó esta especie a la familia Littorinidae basándose en la observación de estas estructuras tentaculares en larvas de Nodilittorina peruviana (Lamarck, 1882) cultivadas en laboratorio (trabajo en preparación) y en la concordancia de la forma de la concha larval y el número de anfractos de los Littorinidae descritos en la literatura (Lebour 1935b, Thorson 1946, Pilkington 1971, Struhsaker \& Costlow 1968, Kowalke 1998b). Cabe destacar el trabajo sobre sistemática del orden Littorinimorpha realizado por Kowalke (1998b) en el cual describe el género Nodilittorina con una concha larval de alrededor de 2,75 anfractos, ornamentación espiral en zigzag (Kowalke 1998b, Figura 8, p. 67 y Figura 4, p. 116), como la observada en los ejemplares de este trabajo, y apertura tipo sinusígera. Este último carácter se presenta en larvas de $N$. peruviana, pero no se observó en los ejemplares aquí descritos, los que presentan un borde ensanchado y recto. En base a las comparaciones anteriores se propone Nodilittorina araucana como la identificación más probable para esta especie.

\section{Collumbellidae}

La morfología de la concha larval de Mitrella sp. coincide con los caracteres de otras especies de la familia. Alrededor de tres anfractos forman la concha larval de Collumbellidae, el anfracto del cuerpo es tres a cinco veces más alto que la espira, la ornamentación es muy reducida y sólo destaca los relieves el canal sifonal (Scheltema 1969, Taylor 1975, Hickman 1999).

Dos de las tres especies de Mitrella descritas para Chile se han citado sólo para la Primera Región. Mitrella unifasciata (Sowerby, 1832) se distribuye de la Primera a la Cuarta Región constituyéndose en la especie más probable a la cual adjudicar la especie Mitrella sp.

Las observaciones de la similitud morfológica en las larvas de las especies Salitra radwini y Mitrella sp. evidencian la importancia de considerar caracteres larvales de la concha y de las partes blandas en el establecimiento de relaciones filogenéticas.

\section{Nassaridae}

La obtención de juveniles a partir de larvas recolectadas es una opción recomendable para lograr una identificación específica. La mantención de ejemplares de Nassarius dentifer postmetamórficos fue fácil y solo requirió del otorgamiento esporádico de alimento fresco para obtener, en un plazo de tres meses, un ejemplar reconocible al nivel de especie. 
La similitud entre ejemplares de Nassarius dentifer y Nassarius sp. y otras especies descritas en la literatura (Lebour 1931a, 1937, Fretter \& Graham 1962, Scheltema 1962, Robertson 1974, Taylor 1975, Hickman 2001), evidencia su relación taxonómica. En base a la distribución geográfica del género, la especie Nassarius sp. podría corresponder a Nassarius gayi (Kiener, 1835) o N. taeniolatus (Philippi, 1860), ambos presentes en la Cuarta Región.

De la literatura revisada para este trabajo, sólo se contó con un estudio de caracterización de larvas de gastrópodos para Chile. El trabajo realizado por Acevedo (1995) utilizó larvas capturadas frente a Quinteros, Quinta Región de Chile y reconoció 19 tipos larvales. Considerando el único ejemplar de Concholepas concholepas recolectado y las 13 especies caracterizadas en este estudio preliminar, se concluye que al menos 5 especies ocurren coincidentemente en ambas regiones. Los tipos larvales AE, F, J, R de Acevedo (1995) corresponden, respectivamente, a las Especies Nassarius dentifer, Salitra radwini, Cerithiidae y Cerithiopsis sp. de este estudio.

Respecto a la forma usual de análisis de plancton, la mayoría de los trabajos sobre taxonomía de larvas de gastrópodos utiliza muestras fijadas luego de su recolección u obtenidas de sedimentos. Estas metodologías presentan dos desventajas importantes. Primero, impiden observar forma, tamaño, pigmentación del velo, del pie y otras partes blandas, estructuras que también representan caracteres taxonómicos. Y en segundo lugar, no se puede determinar el estado de desarrollo larval y en consecuencia, la asignación de un tipo larval a un taxón, puede estar distorsionado o dificultado por la apreciación parcial de la forma tamaño y ornamentación de la concha larval. De esta manera, se hace altamente recomendable iniciar la identificación de larvas de gastrópodos con muestras vivas. Una vez, caracterizadas hasta larvas competentes, los estados intermedios podrán ser reconocidos fácilmente, aún en muestras fijadas.

\section{Proyecciones del estudio}

El estudio de Acevedo (1995) y el presente trabajo son los primeros esfuerzos que se realizan en Chile con el fin de entregar la identificación y caracterización de larvas de un amplio número de especies de gastrópodos. Las proyecciones de este trabajo preliminar son múltiples. Uno de los objetivos abordables en el mediano plazo es la determinación de patrones de abundancia temporales y espaciales, generando la posibilidad de asociar especies a factores bióticos, tales como alimento, depredadores y abióticos como temperatura, salinidad y campos de densidad. Otro aspecto interesante de explorar se relaciona con el valor de las larvas de gastrópodos como indicadores biológicos de contaminación por detergentes, desechos orgánicos metales pesados -particularmente importante considerando el carácter "minero" de Chile -, fenómenos climáticos como "El Niño", corrientes y otros. Los pterópodos son considerados desde hace tiempo como indicadores hidrológicos (Van der Spoel \& Boltovskoy 1981), pero aún no se ha explorado la utilidad de las larvas de gastrópodos en este sentido.

Y por último, la identificación de las larvas de gastrópodos permitirá determinar la ocurrencia de transporte oceánico, como el que se ha establecido para larvas teleplánicas en el Océano Atlántico (Scheltema 1971, 1986, Scheltema \& Williams 1983, Pechenik et al. 1984, Poulin et al. 2002) y la migración desde estados tempranos post-eclosión hasta la etapa competente.

\section{AGRADECIMIENTOS}

Agradecemos a los directores del "proyecto monitoreo regional del impacto de los eventos "el niño" sobre los recursos biológicos y sus usos en América Latina, Riben, Coquimbo, Chile", a los Srs. Enzo Acuña, Julio Moraga y Armando Mujica quienes contribuyeron con las muestras utilizadas en este trabajo. Extendemos nuestros agradecimientos a la dra. Elizabeth von Brand Jefe del proyecto FONDEF No .9811044 por colaborar con la captura de imágenes y al sr. Joel Barraza, Jefe de la unidad de producción de la Universidad Católica del Norte por su colaboración en el mantenimiento de larvas en ambiente controlado.

\section{LITERATURA CITADA}

ACEVEDO C (1995) Caracterización morfológica de larvas de moluscos gastrópodos obtenidas frente a Quintero, V Región. Tesis de Licenciatura en Biología Marina, Instituto de Oceanología, Universidad de Valparaíso, Valparaíso, Chile. $\mathrm{x}+115$ pp.

ALLMON WD (1988) Ecology of recent turritelline gastropods (Prosobranchia, Turritellidae): current knowledge and palaeontological implications. Palaios 3: 259-284.

ALLMON WD (1996) Systematic and evolution of Cenozoic American Turritellidae (Mollusca: Gastropoda) I: paleocene and eocene coastal plain species related to "Turritella mortoni Conrad" and "Turritella humerosa Conrad". Palaeontographica Americana 59: 1-134. 
ALLMON W, D JONES \& N VAUGHAN (1992) Observations on the biology of Turritella gonostoma Valenciennes (Prosobranchia: Turritellidae) from the Gulf of California. Veliger 35: 52-63.

BANDEL K (1976) Observations on spawn, embryonic development, and ecology of some Caribbean lower Mesogastropoda (Mollusca). Veliger 18: 249-271.

BANDEL K \& T KOWALKE (1997a) Eocene Melanotarebia $\mathrm{n}$. g. and its relations among modern Thiaridae (Caenogastropoda: Cerithiodea). Neues Jahrbuch für und Paläontologie 11: 683-695.

BANDEL K \& T KOWALKE (1997b) Systematic value of the larval shell of fossil and modern Vanikoridae, Pickworthiidae and the genus Fossarus (Caenogastropoda, Mollusca). Berliner Geowissenshaftliche Abhandlungen 25: 3-29.

BANDEL K, F RIEDEL \& H WEIKERT (1997) Planktonic gastropod larvae from the Red Sea: a synopsis. Ophelia 47: 151-202.

CAÑETE JI (1992) Cápsulas ovígeras de cinco especies de neogastrópodos de la zona norte de Chile. Boletín de la Sociedad de Biología de Concepción (Chile) 63: 43-49.

COLMAN JG, PA TYLER \& JD GAGE (1986) Larval development of deep-sea gastropods (Prosobranchia: Neogastropoda) from the Rockall Trough. Journal of the Marine Biological Association of the United Kingdom 66: 951-965.

DiSALVO L (1988) Observations on the larval and postmetamorphic life of Concholepas concholepas (Bruguière, 1789) in laboratory culture. Veliger 304: 358-368.

FRETTER V \& A GRAHAM (1962) British Prosobranch Molluscs. Their functional anatomy and ecology. Ray Society of London, Bartholomew Press, London, United Kingdom. 755 pp.

FRETTER V \& M C PILKINGTON (1970) Prosobranchia, veliger larvae of Taenioglossa and Stenoglossa. Fiches d'identification du Zooplancton 129-132: 3-26.

GALLARDO CS (1973) Desarrollo intracapsular de Concholepas concholepas (Bruguiere) (Gastropoda: Muricidae). Museo Nacional de Historia Natural, Santiago, Chile, Publicación Ocasional No. 16. 16 pp.

GALLARDO CS (1977) Two modes of development in the morphospecies Crepidula dilatata (Gastropoda: Calyptraeidae) from southern Chile. Marine Biology 39: 241-251.

GALLARDO CS (1981) Posturas y estado de eclosión del gastrópodo Muricidae Chorus giganteus (Lesson, 1829). Studies on Neotropical Fauna and Environment 16: 35-44.

GALLARDO CS \& K GONZÁLEZ (1994) Oviposturas y desarrollo intracapsular de Xanthochorus cassidiformis (Blainville, 1832) (Gastropoda: Muricidae) de la costa sur de Chile. Gayana Zoología (Chile) 56: 79-90.

HICKMAN CS (1999) Adaptative function of gastropod larval shell features. Invertebrate Biology 118: 346356.

HICKMAN CS (2001) Evolution and development of gastropod larval shell morphology: experimental evidence for mechanical defense repair. Evolution and Development 3: 18-23.
JABLONSKY D \& RA LUTZ (1983) Larval ecology of marine benthic invertebrates: paleobiological implications. Biological Reviews 58: 21-89.

JUNG P (1975) Quaternary larval gastropods from Leg 15, Site 147, deep sea drilling proyect: preliminary report. Veliger 18: 109-126.

KEEN AM (1971) Sea shell of tropical West America. Stanford University Press, Stanford, California. x + $1064 \mathrm{pp}$.

KENNEDY J \& B KEEGAN (1992) The encapsular developmental sequence of the mesogastropod Turritella communis (Gastropoda, Turritellidae). Journal of the Marine Biological Association of the United Kingdom 72: 783-805.

KOOL SP (1993) Phylogenetic analysis of the Rapaninae (Neogastropoda: Muricidae). Malacologia 35: 155259.

KOWALKE T (1998a) Palaeoecology, protoconchmorpholgy and systematic position of the genus Pseudamaura P. Fischer, 1885 (Caenogastropoda: Pseudamauridae). Neues Jahrbuch für und Paläontologie 10: 577-586.

KOWALKE T (1998b) Bewerttung protoconchmorphologischer Daten basaler Caenogastropoda (Cerithiimorpha und Littorinimorpha) hinsichtlich ihrer systematik und evolution von der kreide bis rezent. Berliner Geowissenshaftliche Abhandlungen 27: 1-120.

KOWALKE T (2001) Cerithioidea (Caenogastropoda: Cerithiimorpha) of Tethyan coastal swamps and their relations to modern mangal communities. Bulletin of the Czech Geological Survey 76: 253-271.

KOWALKE T \& K BANDEL (1996) Systematik und Paläoökologie der Küstenschnecken der nordalpinen Brandenberg-Gosau (Oberconiac/Untersanton) mit einem Vergleich zur Gastropodenfauna des Maastrichts des Trempbeckens (Südpyrenäen, Spanien). Mitteilungen der Bayerische Staatssammlung für Paläontologie und Historische Geologie 36: 15-71.

LANCELLOTI DA \& JA VÁSQUEZ (2000) Zoogeografía de macroinvertebrados bentónicos de la costa de Chile: contribución para la conservación marina. Revista Chilena de Historia Natural 73: 99-129.

LEBOUR MV (1931a) The larval stages of Nassarius reticulatus and Nassarius incrassatus. Journal of the Marine Biological Association United Kingdom 17: 797-818.

LEBOUR MV (1931b) The larval stages of Trivia europea. Journal of the Marine Biological Association United Kingdom 17: 819-832.

LEBOUR MV (1932) The larval stages of Simnia patula. Journal of the Marine Biological Association United Kingdom 18: 107-115

LEBOUR MV (1933a) The british species of Trivia: T. arctica and T. Monacha. Journal of the Marine Biological Association United Kingdom 18: 477-484.

LEBOUR MV (1933b) The life-histories of Cerithiopsis tubercularis (Montagu), C. barleei Jeffreys and Triphora perversa (L). Journal of the Marine Biological Association United Kingdom 18: 491-498.

LEBOUR MV (1933c) The eggs and larvae of Turritella communis Lamarck and Aporrhais pes-pelicani (L.). Journal of the Marine Biological Association United Kingdom 18: 499-506. 
LEBOUR MV (1934a) The eggs and larvae of some British Turridae. Journal of the Marine Biological Association United Kingdom 19: 541-510.

LEBOUR MV (1934b) Rissoid larvae as food of the young herring. The eggs and larvae of the Plymouth Rissoidae. Journal of the Marine Biological Association United Kingdom 19: 523-540.

LEBOUR MV (1935a) The larval stages of Balcis alba and $B$. devians. Journal of the Marine Biological Association United Kingdom 20: 65-70.

LEBOUR MV (1935b) The breeding of Littorina neritoides. Journal of the Marine Biological Association United Kingdom 20: 373-378.

LEBOUR MV (1936) Notes on eggs and larvae of some Plymouth prosobranchs. Journal of the Marine Biological Association of the United Kingdom 20: 547-565.

LEBOUR MV (1937) The eggs and larvae of british prosobranch with special reference to those living in the plankton. Journal of the Marine Biological Association of the United Kingdom 22: 105-166.

LEBOUR MV (1945) The eggs and larvae of some prosobranchs from Bermuda. Proceedings of the Zoological Society of London 114: 462-489.

LIMA GM \& RA LUTZ (1990) The relationship of larval shell morphology to the mode of development in marine prosobranch gastropods. Journal of the Marine Biological Association of the United Kingdom 70: 611-637.

MARINCOVICH L (1973) Intertidal mollusks of Iquique, Chile. Natural History Museum, Los Angeles County Science Bulletin 16: 1-49.

MARSHALL BA (1978) Cerithiopsidae (Molusca: Gastropoda) of New Zealand and provisional classification of the family. New Zealand Journal of Zoology 5: 47-120.

MARSHALL BA (1983) A revision of the recent Triphoridae of southern Australia (Mollusca: Gastropoda). Records of the Australian Museum 2: 1119.

MARWICK J (1957) Generic revision of Turritellidae. Proceeding of the Malacological Society of London 32:144-166

NÜTZEL A \& W KIEßLING (1997) Gastropoden aus dem Amaltheeton (oberes Pliensbachium) von Kalchreut. Geologische Blätter NO-Bayern 47: 381-414.

NÜTZEL A (1998) Über die Stammesgeschichte der Ptenoglossa (Gastropoda). Berliner Geowissenshaftliche Abhandlungen 26: 1-229.

PECHENIK JA, RS SCHELTEMA \& LS EYSTER (1984) Growth stasis and limited shell calcification in larvae of Cymatium parthenopeum during trans-Atlantic transport. Science 224: 1091-1096.

PILKINGTON M (1971) Eggs, larvae, and spawning in Melarpha cincta (Quoy \& Gaimard) and M. oliveri Finlay (Littorinidae, Gastropoda). Australian Journal of Marine Freshwater Research 22: 79-90.

POULIN E, AT PALMA, G LEIVA, E HERNÁNDEZ, P MARTÍNEZ, SA NAVARRETE, JC CASTILLA (2002) Temporal and spatial variation in the distribution of epineustonic competent larvae of Concholepas concholepas along the central coast of Chile. Marine Ecology Progress Series 229: 95-104.
RAMÍREZ J (1987) Católogo: moluscos de Chile, Volumen II: Mesogastropoda. Publicación artesanal, Santiago, Chile. 194 pp.

RAMÍREZ J (1999) Catálogo: moluscos de Chile, Volumen III: Neogastropoda. Publicación artesanal, Santiago, Chile. 168 pp.

ROBERTSON R (1971) Scanning electron microscopy of planktonic larval marine gastropod shell. Veliger 14: $1-12$

ROBERTSON R (1974) Marine prosobranch gastropods: larval studies and systematics. Thalassia Jugoslavica 10: $215-238$

RODRÍGUEZ C \& C THIRIOT-QUIEVREUX (1974) Gastéropodes de la région de Roscoff-étude particulière de la protoconque. Cahiers de Biologie Marine 15: 531-549.

RODRÍGUEZ C \& C THIRIOT-QUIEVREUX (1975) Pyramidellidae, Philinidae et Retusidae de la région de Roscoff. Etude pariculière des protoconques de quelques espèces. Cahiers de Biologie Marine 16: 8396.

ROMERO M (1995) Desarrollo intracapsular, extracapsular, asentamiento y metamorfosis de Thais (Stramonita) chocolata (Duclos, 1832), en condiciones de laboratorio. Tesis de Licenciatura, Facultad de Ciencias del Mar, Universidad Católica del Norte, Coquimbo, Chile. 155 pp.

ROMERO MS, K LOHRMANN, G BELLOLIO \& E DUPRE (en prensa) Comparative observations on reproductions, spawning, and early veliger of three common subtidal mesogastropods from north-central Chile. The Veliger.

SCHELTEMA AH (1969) Pelagic larvae of New England gastropods. IV Anachis translirata and Anachis avara (Collumbellidae, Prosobranchia). Vie et Milieu 20: 94-104.

SCHELTEMA RS (1962) Pelagic larvae of New England intertidal gastropods I. Nassarius obsoletus Say and Nassarius vibex Say. Transactions of the American Microscopical Society 81: 1-112.

SCHELTEMA RS (1966) Evidence for trans-Atlantic transport of gatropod larvae belonging to the genus Cymatium. Deep-Sea Research 13: 83-95.

SCHELTEMA RS (1971) Larval dispersal as a means of genetic exchange between geographically separated populations of shallow-water benthic marine gastropods. Biological Bulletin 140: 284-322.

SCHELTEMA RS (1979) Dispersal of pelagic larvae and the zoogeography of Tertiary Marine Benthic Gastropods. En: Gray J \& AJ Boucot (eds) Historical biogeography, plate tectonics, and the changing enviroment: 391-397. Oregon State University Press, Corvallis, Oregon.

SCHELTEMA RS (1986) On dispersal and planktonic larvae of benthic invertebrates: an eclectic overview and summary of problems. Bulletin of Marine Science 39: 290-322.

SCHELTEMA RS \& IP WILLIAMS (1983) Long-distance dispersal of planktonic larvae and the biogeography and evolution of some polynesian and western pacific mollusk. Bulletin of Marine Science 33: 545-565.

SEAPY R \& C THIRIOT-QUIEVREUX (1994) Veliger larvae of Carinariidae (Mollusca: Heteropoda) from hawaiian waters. Veliger 37: 336-343. 
STRUHSAKER J \& J COSTLOW (1968) Larval development of Littorina picta (Prosobranchia, Mesogastropoda), reared in laboratory. Proceedings of the Malacological Society of London 38: 153-160.

TAYLOR J (1975) Planktonic prosobranch veligers of Kaneohe Bay. Doctoral Dissertation, University of Hawaii, Honolulu, Hawaii. 593 pp.

THIRIOT-QUIÈVREUX C (1967a) Descriptions de quelques veligeres planctoniques de gasteropodes. Vie et Milieu 2: 303-315.

THIRIOT-QUIÈVREUX C (1967b) Observations sur le développement larvaire et postlarvaire de Simnia spelta Linné (Gastèropode Cypraeidae). Vieu et Milieu 1: $143-151$.

THIRIOT-QUIÈVREUX C (1968) Variations saisonnières des mollusques dans le plancton de la région de Banyuls-sur-Mer (zone sud du golfe du Lion) Novembre 1965-décembre 1967. Vie et Milieu 19: 35-83.

THIRIOT-QUIÉVREUX C (1969) Caractéristiques morphologiques des véligères planctoniques de gastéropodes de la région de Banyuls-Sur-Mer. Vie et Milieu 20: 333-366.

THIRIOT-QUIÉVREUX C (1970) Cycles annuels des populations plantoniques de mollusques en 1968 dans la région de Banyuls-Sur-Mer. Comparaison avec les années précédentes 1965-1967.Vie et Milieu 21:311336.

THIRIOT-QUIÉVREUX C (1972) Microstructures de coquilles larvaires de Prosobranches au microscope électronique à balayage. Archives de Zoologie Expérimentale et Générale 113: 553-564.

THIRIOT-QUIÉVREUX C (1974) Anatomie interne de véligères planctoniques de prosobranches mésogastropodes au stade proche de la métamorphose. Thalassia Jugoslavica 10: 379-399.

THIRIOT-QUIÉVREUX C (1975) Observations sur les larves et les adultes de Carinariidae (Mollusca: Heteropoda) de 1'Ocean Atlantique Nord. Marine Biology 32: 379-388.
THIRIOT-QUIÈVREUX C (1977) Véligère planctotrophe du doridien Aegires punctilucens (D’Orbigny) (Mollusca:Nudibranchia:Notodorididae): desciption et metamorphose. Journal of Experimental Marine Biology and Ecology 26: 177-190.

THIRIOT-QUIÈVREUX C (1980) Identification of some planktonic prosobranch larvae present off Beaufort, North Carolina. Veliger 23: 1-9.

THIRIOT-QUIÈVREUX C (1980) Summer meroplanktonic prosobranch larvae occurring off Beaufort, North Carolina. Estuaries 6: 387-398.

THIRIOT-QUIÈVREUX C \& C RODRÍGUEZ (1975) Etude des protoconques de quelques prosobranches de la région de Roscoff. Cahiers de Biologie Marine XVI: $135-148$

THIRIOT-QUIÈVREUX C \& R SCHELTEMA (1982) Planktonic larvae of New England gastropods, $V$. bittium alternatum, Triphora nigronicta, cerithiopsis emersoni, Lunatia heros and crepidula plana. Malacologia 23: 37-46.

THORSON G (1946) Reproduction and larval development of Danish marine bottom invertebrates, with special reference to the planktonic larvae in the sound (Oresund). Meddelelser fra Kommisionen for Danmarks Fiskeri-og Havundersmogelser, Copenhagen, Serie Plankton 4: 1-527.

THORSON G (1950) Reproductive and larval ecology of marine bottom invertebrates. Biological Reviews 25: $1-45$.

THORSON G (1965) A neotenous dwarf-form of Capulus ungaricus (L.) (Gastropoda, Prosobranchia) commensalistic on Turritella communis Risso. Ophelia 2: 175-210.

VAN DER SPOEL S \& D BOLTOVSKOY (1981) Pteropoda. En: Boltoskoy D (ed) Atlas del zooplancton del Atlántico sudoccidental y método de trabajo con el zooplancton marino: 493-517. Publicación Especial del INIDEP, Mar del Plata, Argentina.

Editor Asociado: S. Navarrete

Recibido el 25 de septiembre de 2001; aceptado el 9 de mayo de 2002 